\title{
Assembly Manual for the Berkeley Lab Cosmic Ray Detector
}

By Michael Collier*

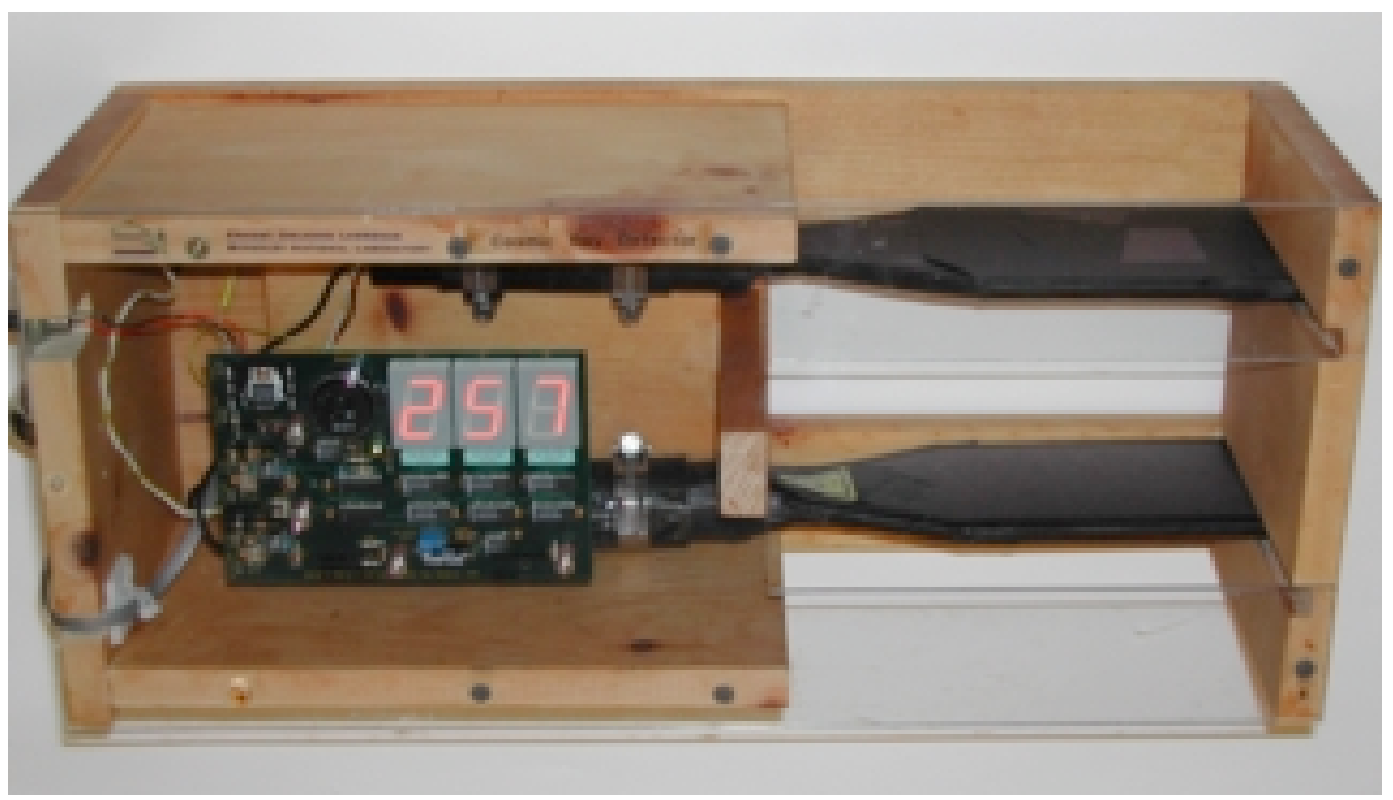

\section{Version 1.1 - December 2002}

*For correspondence contact - Howard Matis - hsmatis@lbl.gov 

Introduction

Scintillator

Cutting 4

Equipment and Supplies 4

Procedure

Lucite Cookies (optional)

Equipment and Supplies

Procedure

Polishing

Equipment and Supplies 10

Procedure - Coarse (600 grit) 10

Procedure - Medium ( $\sim 800$ grit $) \_12$

Procedure — Fine $(\sim 1200$ grit $) \_12$

Gluing _ـ 14

Equipment and Supplies _ 14

Procedure _ـ 14

Wrapping 18

Equipment and Supplies 18

Procedure 18

Circuit Board _ 22

Soldering 22

Equipment and Supplies _ 22

Procedure _L 24

Casing 30

Wiring__ 32

Equipment and Supplies 32

Procedure __ 32

Appendix A: Suppliers and Parts __ 34

Scintillator Paddles__ 34

Circuit Board —_ 34

Appendix B: Resistor Color Codes _ 36

Appendix C: Optional Dimensions for Casing___ 37

Appendix D: Acknowledgements__ 39 


\section{Cosmic Ray Detector}

\section{Introduction}

The Berkeley Lab Cosmic Ray Detector consists of 3 main components that must be prepared separately before they can be assembled. These components are the scintillator, circuit board, and casing. They are described in the following main sections, which may be completed in any order.

\section{Scintillator}

Preparing the scintillator paddles (Figure 1) involves several steps - cutting the scintillator material to the appropriate size and shape, preparing and attaching Lucite cookies (optional), polishing the edges, gluing the end to the photomultiplier tube (optional), and wrapping the scintillator. Since the detector has 2 paddles, each of the following sections needs to be repeated for the other paddle.

\section{Cutting}

The scintillator material must be cut to the appropriate shape to fit onto the photomultiplier tube correctly. Although professionals cut and mill scintillator carefully with special equipment, for our purpose, a regular saw is sufficient. The saw can be a table saw, band saw, jigsaw, or even a hacksaw, but it should be one with a blade that can get wet.

Generally, people handling scintillator should wear gloves to extend the life of the material; the oils from skin deteriorate the scintillator surface over time. However, our detector can tolerate such deterioration.

\section{Equipment and Supplies}

- Scintillator sheet (1/4" thick)

- Film marker (or ink pen)
- Saw (medium spaced teeth)

- Cool water

\section{Procedure}

1. Mark the scintillator sheet with a film marker (or ink pen) according to the dimensions in Figure 2. The manufacturer's paper cover may be left on at this time.

2. Cut the scintillator sheet with a saw along the lines drawn in step 1. Be sure to cut along the outer edge of the line; the excess will be sanded away in the polishing process.

3. If your saw is not continuously wet (drawing water from a tray), then stop to wet it periodically. The water is to keep the scintillator from melting onto the blade.

\section{Remove the paper cover, if you have not already.}




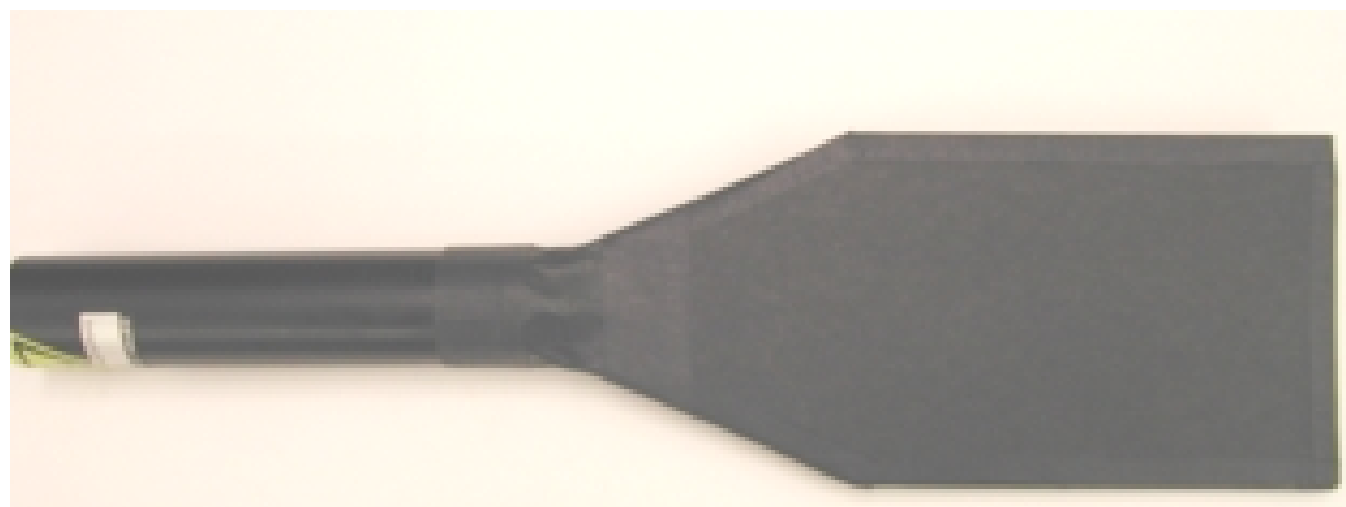

Figure 1: Scintillator paddle completed.
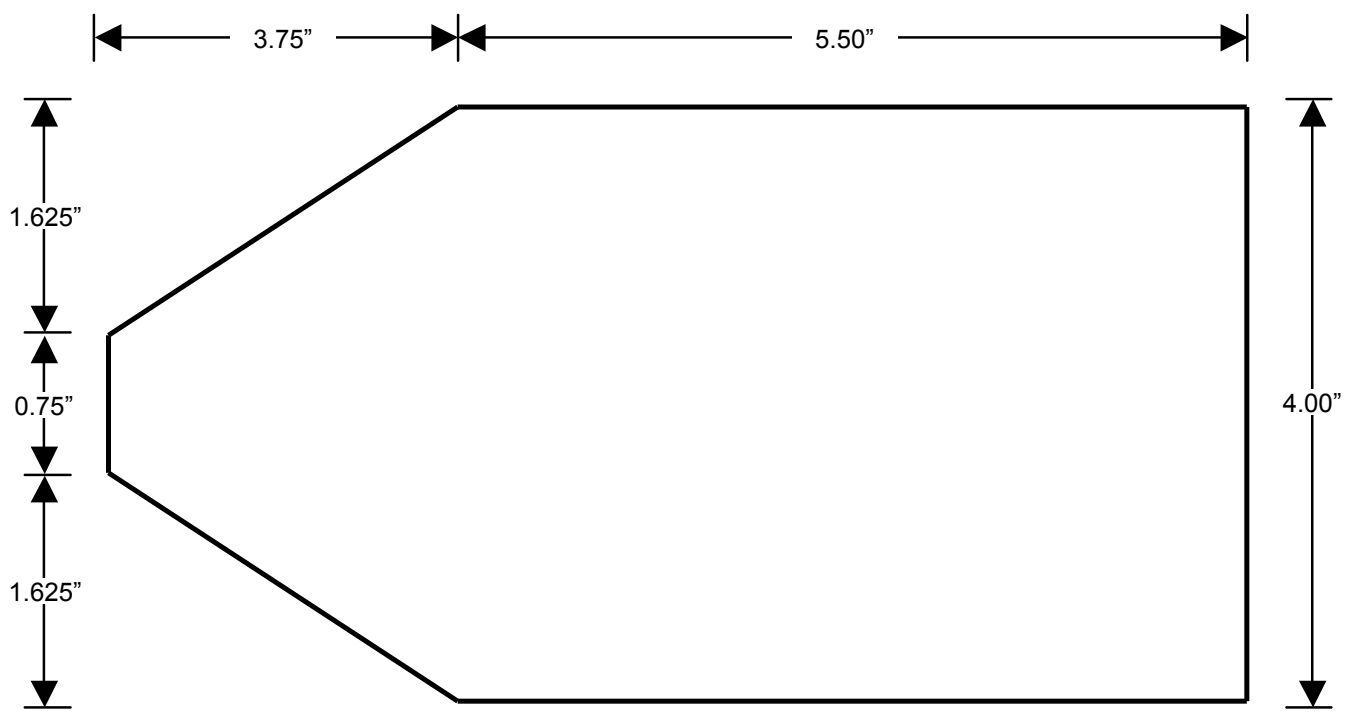

Figure 2: Dimensions for cutting scintillator material. 


\section{Cosmic Ray Detector}

\section{Lucite Cookies (optional)}

The Lucite cookies (Figure 3) attach to the end of the scintillator (the end that will be joined with the photomultiplier tube). They fill in the extra space not taken by the scintillator end on the photomultiplier. They are helpful only if you plan to glue the scintillator to the photomultiplier; they provide a broader gluing surface. Because of this purpose, the cookies do not even need to be Lucite; any plastic even left over scintillator - is sufficient. However, try to find a material that will absorb a minimum of light (that is, a black material is not a good choice). Preparing the cookies involves several steps similar to preparing the scintillator itself: cutting, gluing, and polishing. The cookies may be attached to the scintillator before of after it has been polished.

Cutting Lucite or other plastic is much like cutting the scintillator except that Lucite or other plastic is not as fragile and may be handled more roughly. To minimize the amount of cutting, choose a cylinder or disk of plastic that is $3 / 4$ of an inch in diameter. Otherwise, for example, if you use leftover scintillator, you will have to cut out 2 semicircles (the photomultiplier end is circular with a diameter of about 1 inch).

Gluing the cookies is much like gluing the scintillator to the photomultiplier end. You may want to use the same optical cement just to practice mixing and applying it, but any strong adhesive for plastics will work.

Polishing the cookies is a matter of preference. The surfaces that are glued to the scintillator should be at least smoothed with coarse sandpaper (600 grit). The surfaces that attach to the photomultiplier end will be polished with the rest of the scintillator edges (next section) unless you attach the cookies after polishing the scintillator.

\section{Equipment and Supplies}

- Lucite or plastic (3/4 inch diameter cylinder or disk preferred)

- Saw (as in Cutting section)

- Sandpaper (600 grit)
- Adhesive for plastic (optical cement preferred)

- Film marker (or ink pen)

- Clean cloth or tissue

\section{Procedure}

1. Mark the plastic cylinder crosswise for a $1 / 2$ " thick disk. If you have some other shape of material, then mark 2 semicircles with a radius of $3 / 8$ of an inch.

2. Cut the plastic along the marks. See Figure 4 for illustration. 


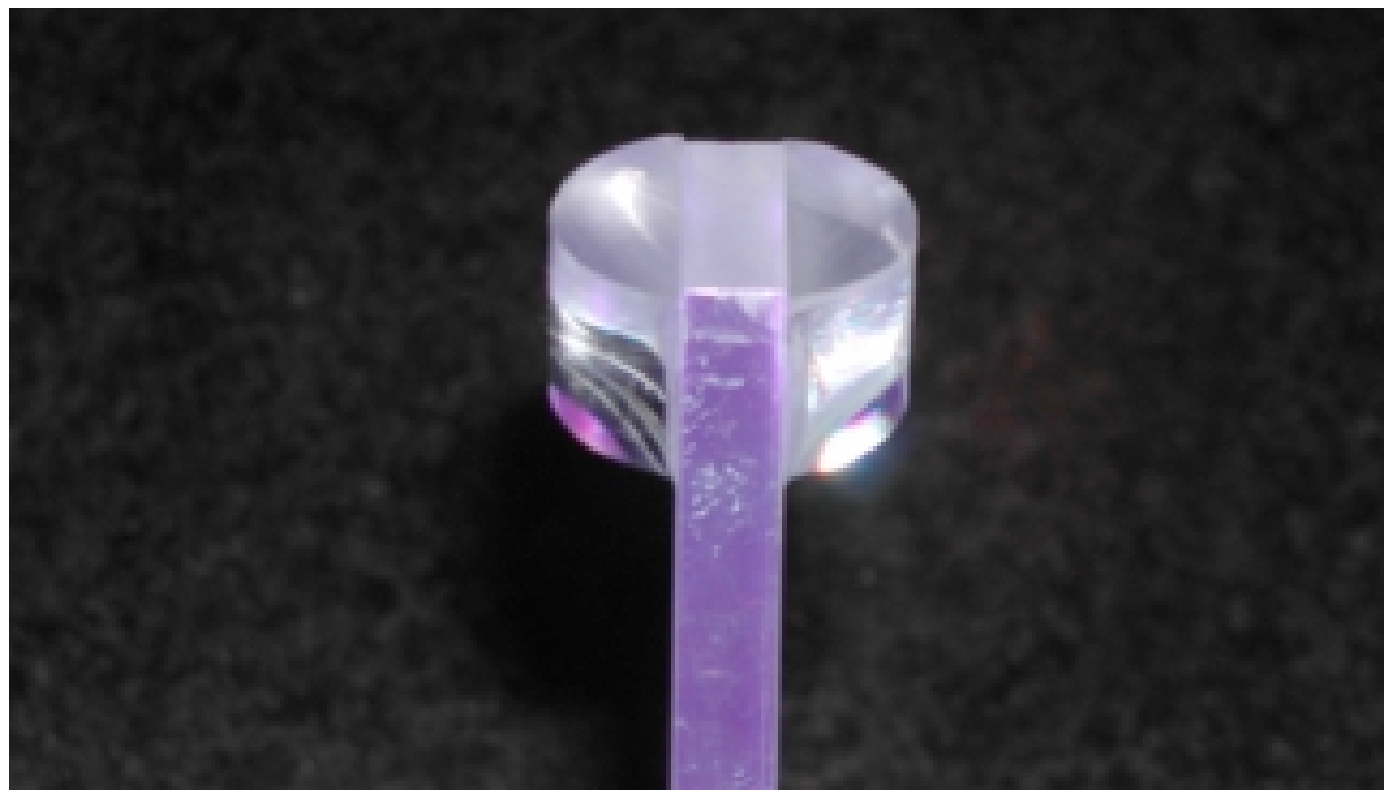

Figure 3: Lucite cookies (half cylinders) attached to 3/4-inch end of scintillator (center strip).

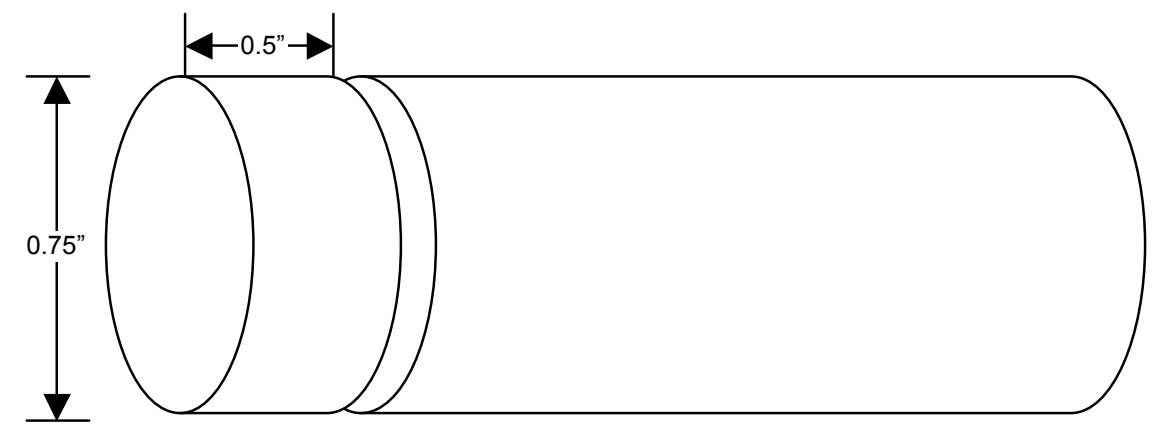

Figure 4: Cylinder of Lucite (or other plastic) showing dimensions for cut. 


\section{Cosmic Ray Detector}

3. If you are using a cylinder, make an additional straight cut through the center of the disk from step 2 (dashed line in Figure 5).

4. Smooth the base edge of the semicircles with 600 grit sandpaper. Follow the same method described in the Coarse Polishing section. Be sure to wipe or wash the sanded surfaces.

5. Check to make sure that the cookies, when attached to the scintillator, will fit on the photomultiplier end. If they are too big, then trim and smooth as appropriate.

6. Prepare the adhesive according to the manufacturer's instructions. See the Gluing section if you are using the optical cement.

7. Glue the bases of each semicircle wing to the top and bottom surfaces of the scintillator at its 3/4-inch edge. Be sure to glue the cookies such that their semicircular flat surfaces are flush against the 3/4-inch edge of the scintillator (Figure 3). This is especially important if you are gluing the cookies after polishing the scintillator.

8. Let the winged scintillator sit undisturbed until the adhesive has hardened or dried. You may need to brace the pieces together in some way while the adhesive sets. 


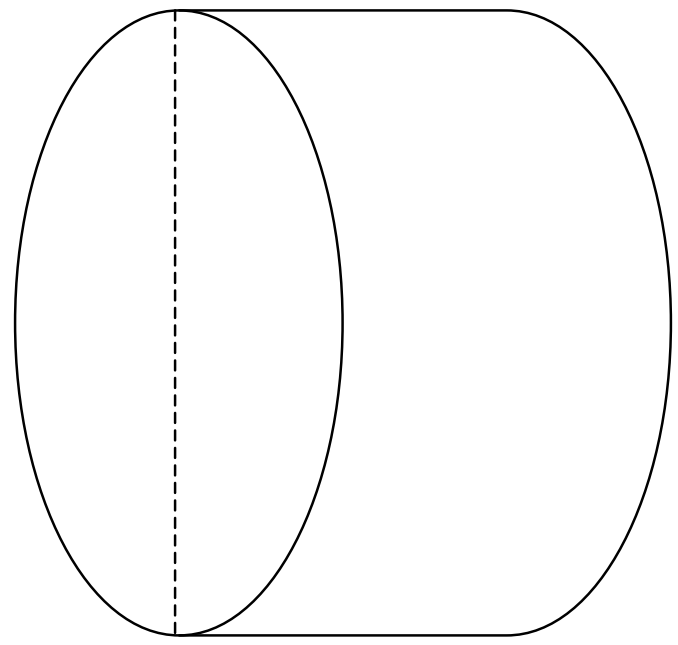

Figure 5: Disk of Lucite (or other plastic) with dashed line to show cut. 


\section{Cosmic Ray Detector}

\section{Polishing}

In order for the very faint flashes of light from cosmic rays to reach the photomultiplier tube, the interface between the tube and scintillator end as well as the other edges must be very transparent. For that reason, the scintillator edges must be polished. (The broad top and bottom surfaces do not need to be polished.) Polishing includes 3 levels - coarse, medium, and fine.

\section{Equipment and Supplies}

- Scintillator cut to shape

- Flat, solid surface

- Putz Pomade

- Rotating pad (if available)

- Alumina powder

- Felt

- Clean cloth or tissue

- Sandpaper (600 grit)

- Cool and warm water

\section{Procedure - Coarse (600 grit)}

1. Break a piece of $\mathbf{6 0 0}$ grit sandpaper. That is, holding 2 opposite ends, pull the paper firmly across a straight metal or stone edge (such as a stainless steel sink or stone countertop) as if polishing a shoe with a rag. This takes out the uneven roughness of the paper.

2. Moisten the sandpaper with clean water. The water will keep the plastic material from filling the pits and valleys in the paper. You may want to periodically re-wet the paper to clean it while sanding.

3. Place the piece of sandpaper on a solid, flat surface with the sand side facing up.

4. Firmly hold the edge of the scintillator flat against the sand paper and push along it in a straight path until you reach the end of the paper. See Figure 6 for illustration.

5. Stop, lift up the scintillator edge, and return it to the starting position.

6. Repeat steps 4 and 5 until the scintillator edge is flat and evenly sanded. The scintillator edge should have grooves or striations all going the same direction and continuous across the surface; it should have no glossy areas. See Figure 7 for illustration.

7. Rinse the scintillator with clean, cool water and dry with a clean cloth or tissue.

Note: Be sure to follow the same direction on each stroke when sanding. This will ensure even sanding. You should also vary the path along the sand paper to maintain sanding effectiveness. 


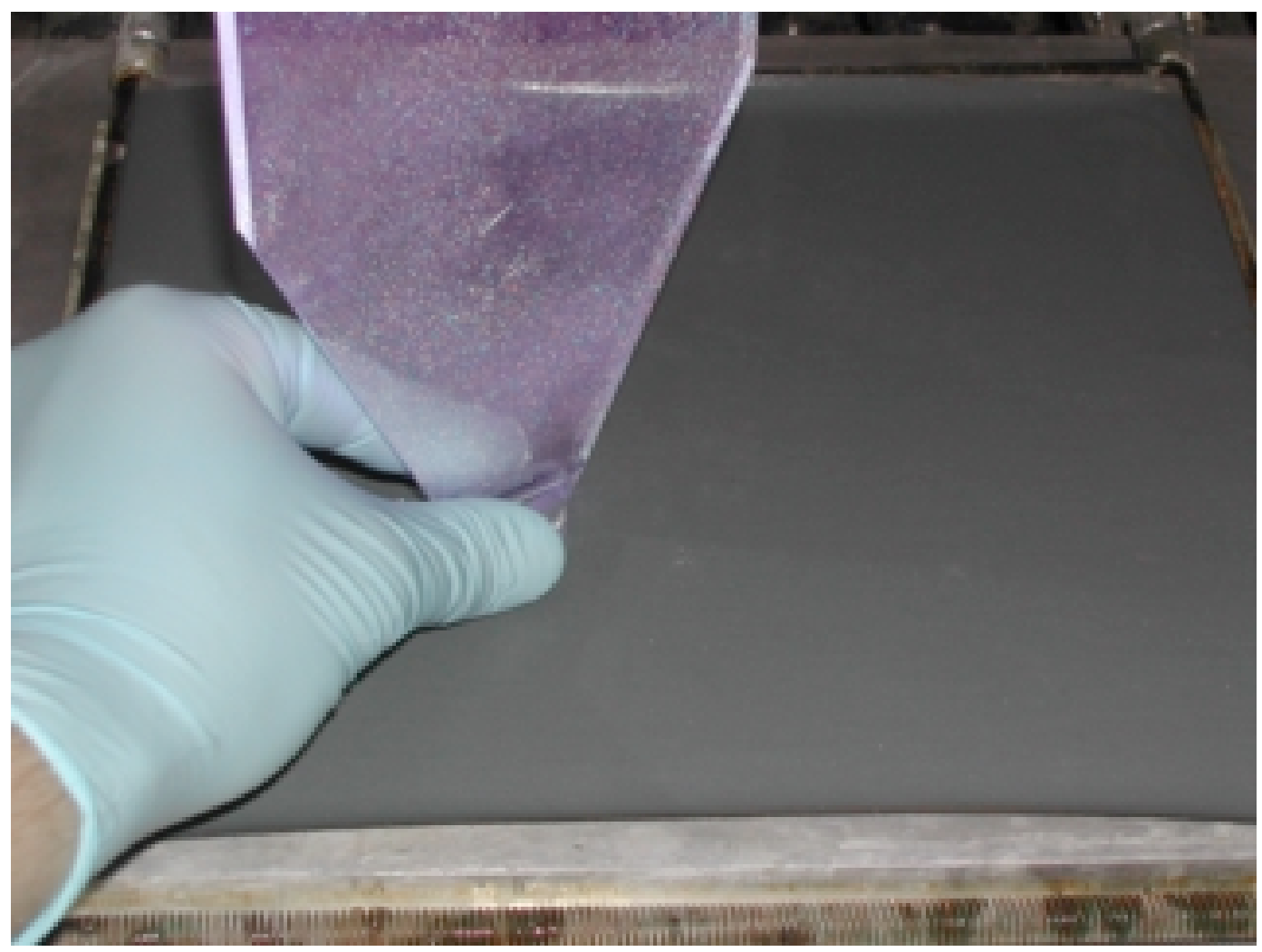

Figure 6: Scintillator end being coarsely sanded.

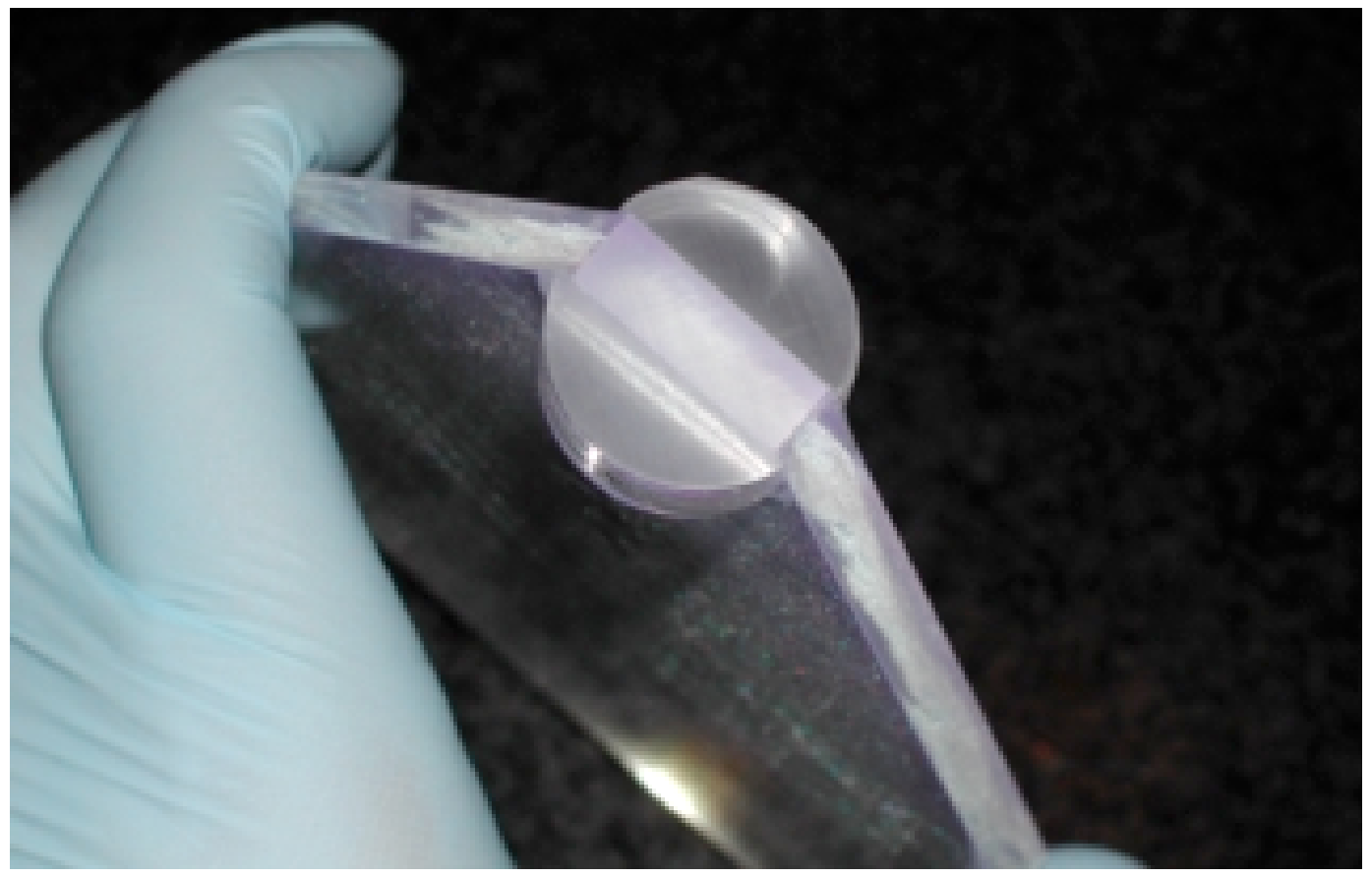

Figure 7: Coarse surface of scintillator. 


\section{Cosmic Ray Detector}

\section{Procedure - Medium ( $>800$ grit)}

1. Cover a rotating pad machine with felt. See Figure 8 for illustration. If you do not have such a machine, then just attach the felt to a flat surface as was done for the sandpaper. The polishing will be just more tedious.

2. Sprinkle some Putz Pomade ( $\sim 800$ grit) onto the felt. The red material in Figure 8 is the putz. If you do not have a rotating pad, go on to step 5.

3. Power the rotating pad machine.

4. Lightly hold the scintillator edge flat against the rotating pad until polished. Be sure to polish against the grain or striations made by the sandpaper. The edge is finished for this step when the Putz has polished away the sandpaper's grain. This may take 1-10 minutes for each edge, depending on your skill.

5. If not using a table, polish in the same manner as with the sandpaper. That is, hold the edge flat against the felt and push in straight lines against the grain made by the sandpaper. The edge is finished for this step when the Putz has polished away the sandpaper's grain. This may take 5-15 minutes for each edge, depending on your skill.

6. Wash the scintillator of the Putz Pomade and dry with a clean cloth or tissue. Mild detergent and water should be used for washing.

\section{Procedure - Fine ( 1200 grit)}

1. Use a fresh piece of felt on the rotating pad machine or flat surface.

2. Sprinkle the felt with alumina powder.

3. Moisten the powdered felt with warm water.

4. Polish according to the steps for the Putz. Polishing with the alumina powder takes about the same amount of time as for the Putz.

5. The scintillator edge is polished when it has no visible grain or striations and is very glossy. See Figure 9. 


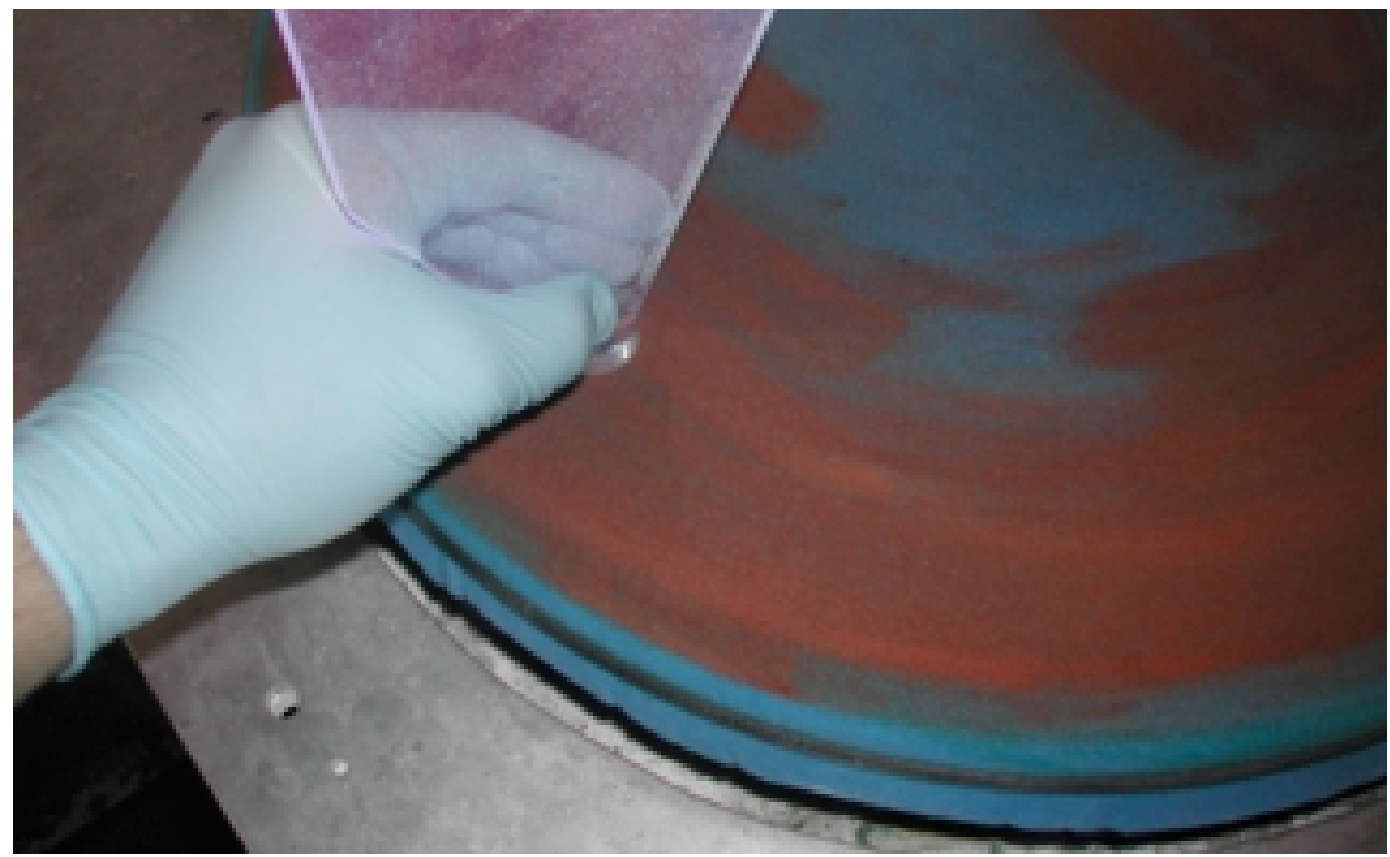

Figure 8: Scintillator being polished with putz on rotating round pad.

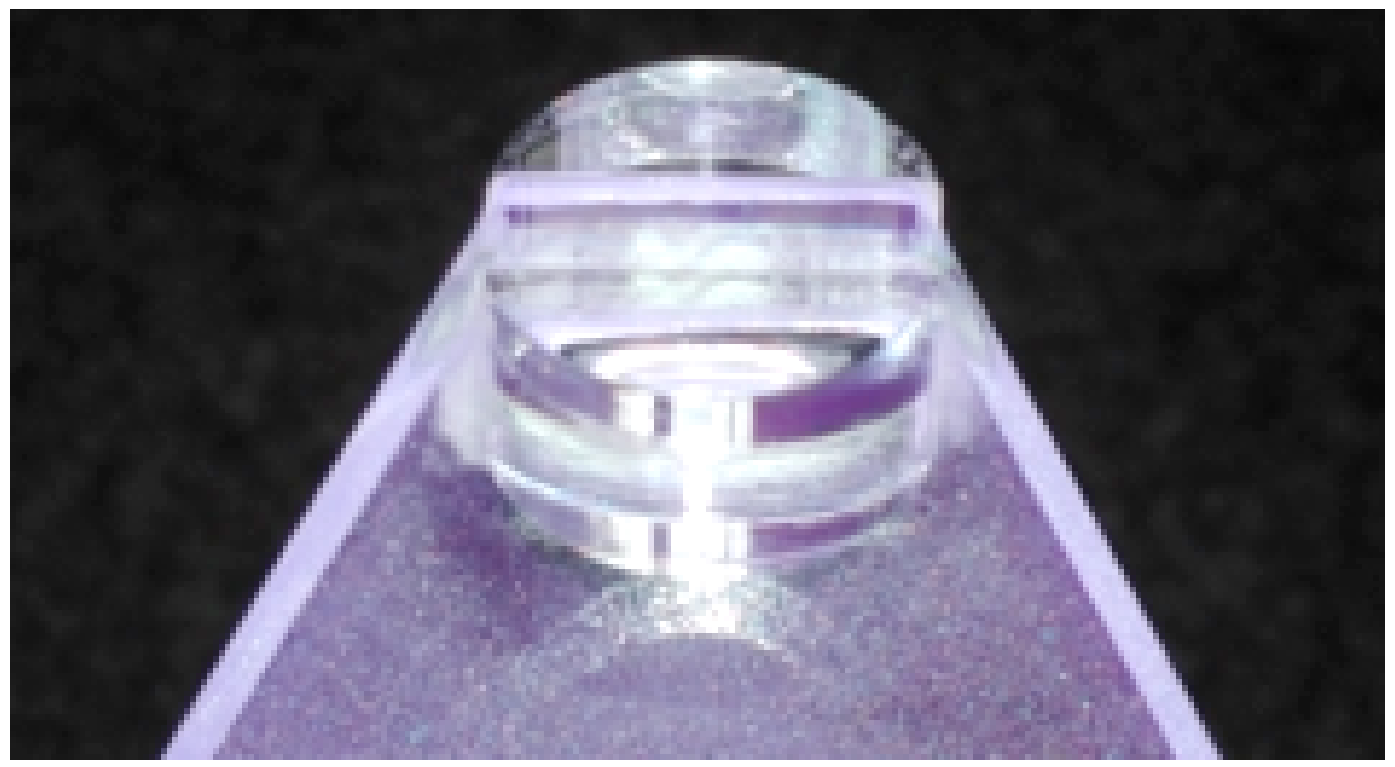

Figure 9: Polished surface of scintillator. 


\section{Cosmic Ray Detector}

\section{Gluing}

For a better detector, the semi-polished scintillator end should be glued to the glass of the photomultiplier tube with optical cement. The optical cement serves not only to bond the scintillator to the photomultiplier but also to reduce the differences in the indices of refraction between the 2 interfaces. The index of refraction for scintillator is typically 1.58 , while it is typically 1.52 for glass. Air has a refractive index of nearly 1, though. Having light pass from a medium of high index to a medium of such low index causes the light to diverge away from the original path, which means that light will escape from the perimeter of the gap between the scintillator and the photomultiplier. The optical cement, with a refractive index of 1.57, minimizes such loss.

However, for our purpose, the optical cement is more important for holding the pieces of the scintillator paddle together. The flashes of light caused by cosmic rays are bright enough to make the losses negligible. Therefore, the optical cement is optional though preferred.

If you decide to not glue the scintillator and photomultiplier together, then continue on to the next section - Wrapping.

\section{Equipment and Supplies}

- Scintillator

- Photomultiplier tube

- Stand for tubes (optional)
- Optical cement

- Lens cloth

\section{Procedure}

1. Mix the optical cement components, if necessary. Follow the manufacturer's instructions for mixing the cement.

2. Let the optical cement mixture sit, if necessary, for $\mathbf{1 5}$ minutes. Sitting allows the air bubbles to escape the mixture. The time may vary among different brands of cement; follow the manufacturer's instructions. Alternatively, you may vacuum the air bubbles out in a small vacuum chamber designed for the purpose (a household vacuum cleaner will not work).

3. Meanwhile, place the photomultiplier tube onto the stand such that the glass end is facing up. If you do not have a stand (as shown in Figure 10), then use some improvised method to hold the photomultiplier with its glass side up (and keep it held for 24-48 hours undisturbed).

4. Clean the surfaces to be glued - the glass of the photomultiplier tube and the polished end of the scintillator - with the lens cloth. Follow any additional instructions provided by the manufacturer of the optical cement for surface preparation. 


\section{Assembly Manual}
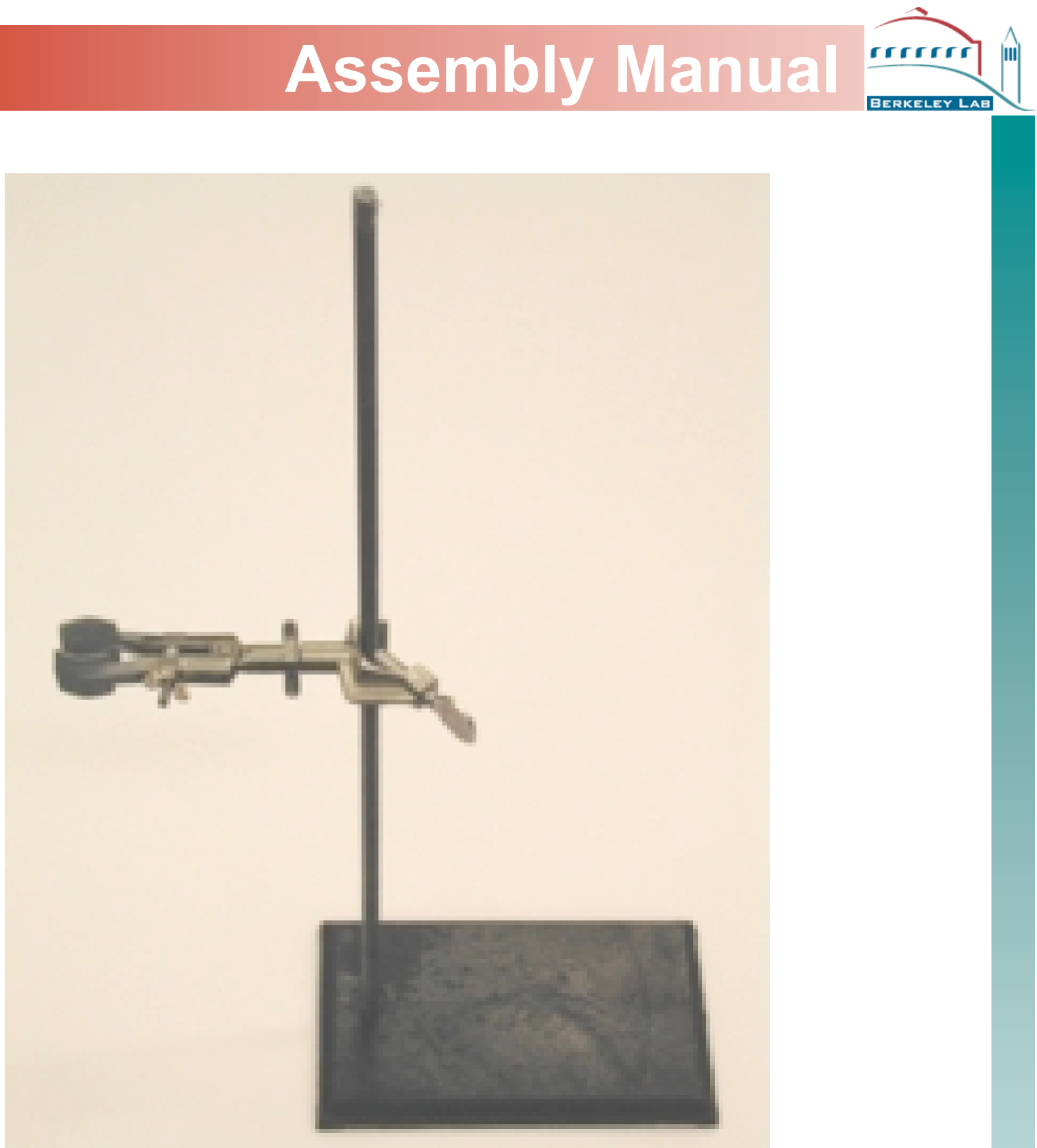

Figure 10: Stand to hold photomultiplier and scintillator. 


\section{Cosmic Ray Detector}

5. Place a couple drops of the settled optical cement onto the glass of the photomultiplier. Be careful that the cement does not drip down the sides. If your photomultiplier does not have a lip on its edge, you may want to make a temporary one with some tape.

6. Set the scintillator end carefully onto the cement and glass of the photomultiplier. Be sure to not trap any air between the surfaces. You may want to place one edge down first and gently lower the other edges - like closing a hinged lid — to squeeze out the air.

7. Let the scintillator and photomultiplier sit undisturbed (Figure 11) for 24-48 hours or the length of time specified by the manufacturer of your optical cement. You may brace the scintillator resting on top of the photomultiplier if necessary. Do not lift the scintillator end off the glass unless you plan to clean the surfaces again and re-glue. 


\section{Assembly Manual}
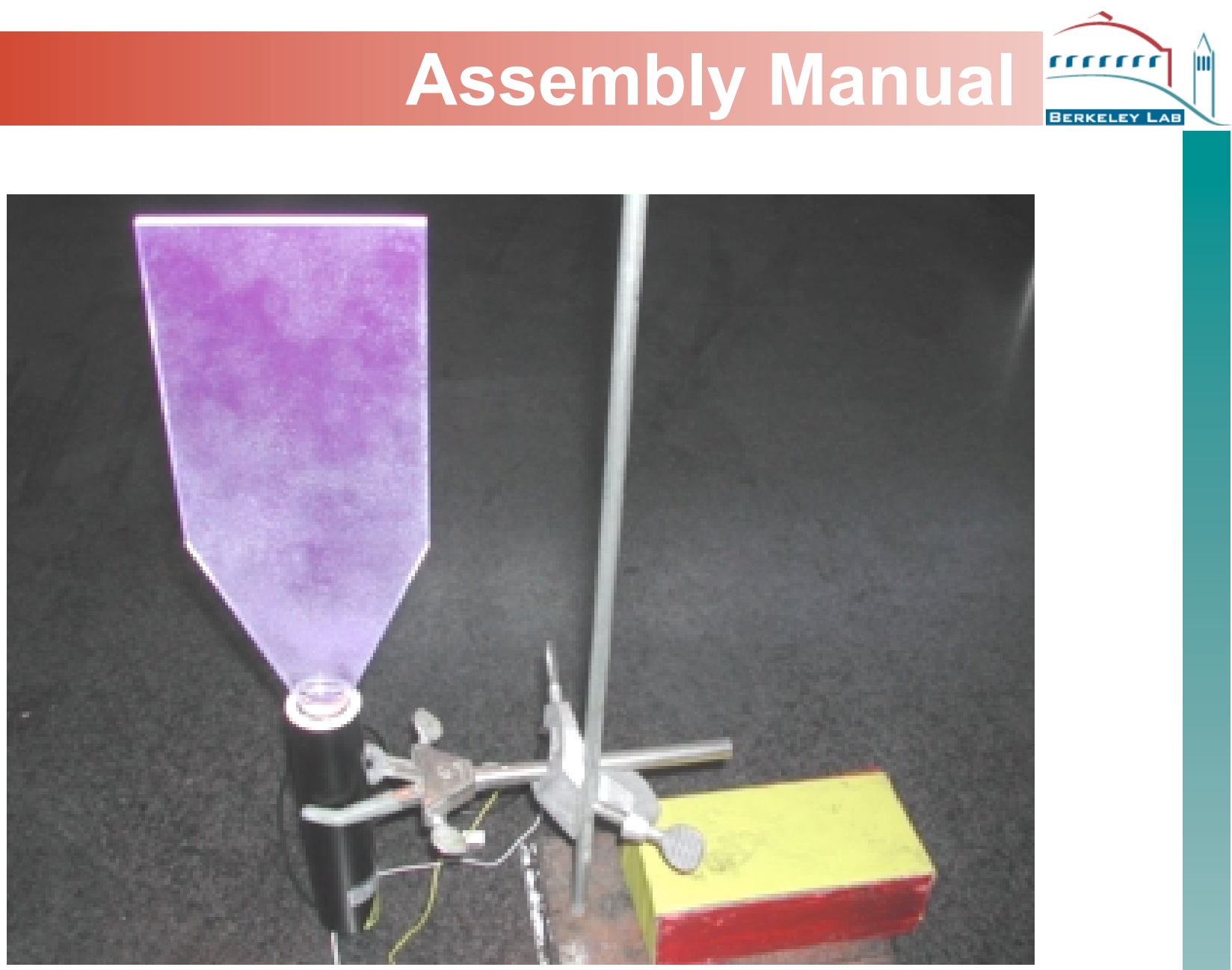

Figure 11: Scintillator bonding with photomultiplier undisturbed for 24-48 hours. 


\section{Cosmic Ray Detector}

\section{Wrapping}

Wrapping the scintillator paddle with opaque materials ensures that the photomultiplier tube detects only the flashes of light produced by cosmic rays. Also, wrapping the scintillator with the photomultiplier provides mechanical support.

\section{Equipment and Supplies}

- Scintillator paddle

- Photomultiplier tube (if not joined to scintillator)

- Aluminum foil (heavy duty)

- Black cardstock
- Black masking tape

- Thin tape (e.g., Scotch)

- Pencil

- Scissors (or utility knife)

\section{Procedure}

1. Mark 2 pieces of black cardstock according to the dimensions in Figure 2. The dimensions are the same as those for the scintillator, but the cardboard should be a pencil line smaller.

2. Cut the cardstock along the marks made in step 1. Be sure to cut just slightly smaller than the dimensions of the scintillator.

3. Set the cardstock pieces aside and lay a sheet of aluminum foil out on a clean, flat surface with the shiny side up.

4. Place the scintillator in the center of the foil and neatly fold the foil over the surfaces and edges of the scintillator. Be sure that the wrapping is tight, like a birthday present (Figure 12).

Note: Keep the aluminum foil away from the scintillator end that attaches to the photomultiplier. The end of the photomultiplier is run at high voltage. Care must be made to prevent any possible electrical contact. It must be insulated. Wrap the foil to just the surface of the Lucite cookies (if you are using cookies).

5. Tape the foil (with the thin tape) to keep it tightly wrapped (Figure 12).

6. Place a small piece of thin tape curled back on itself onto each of the top and bottom surfaces of the scintillator (Figure 13). This curl of tape is to hold the pieces of cardstock in place when trying to seal the edges of the scintillator with the black masking tape.

7. Now stick a piece of the cut black cardstock onto each of the top and bottom surfaces of the scintillator. Be careful to fit the cardstock so that a sliver of space shows between it and the scintillator edges. This space will allow the black masking tape to make good contact with the edges. 


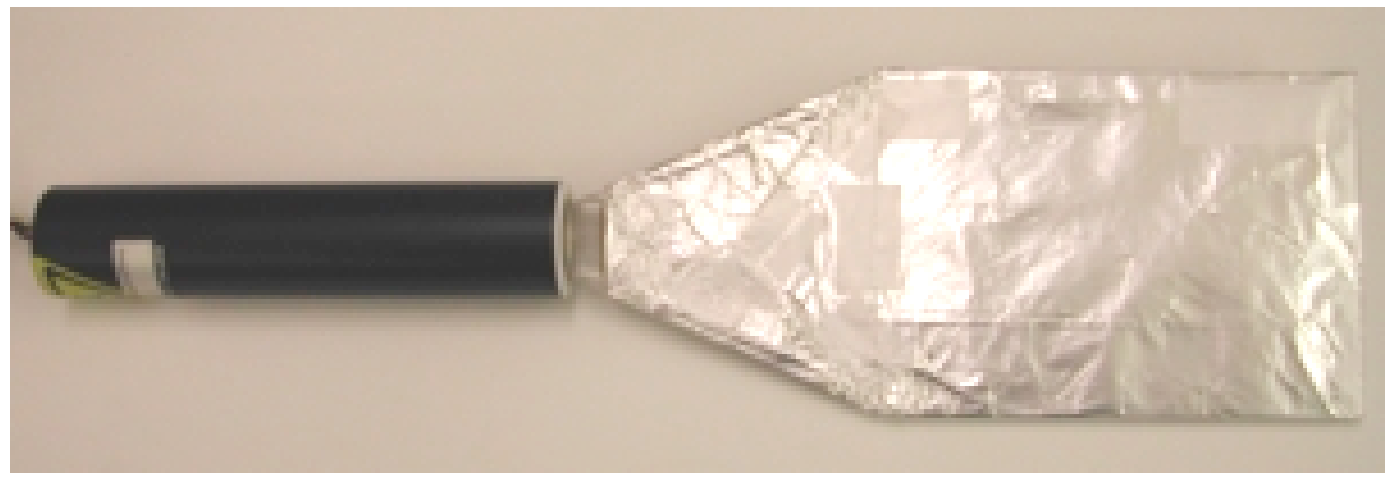

Figure 12: Scintillator paddle wrapped with aluminum foil.

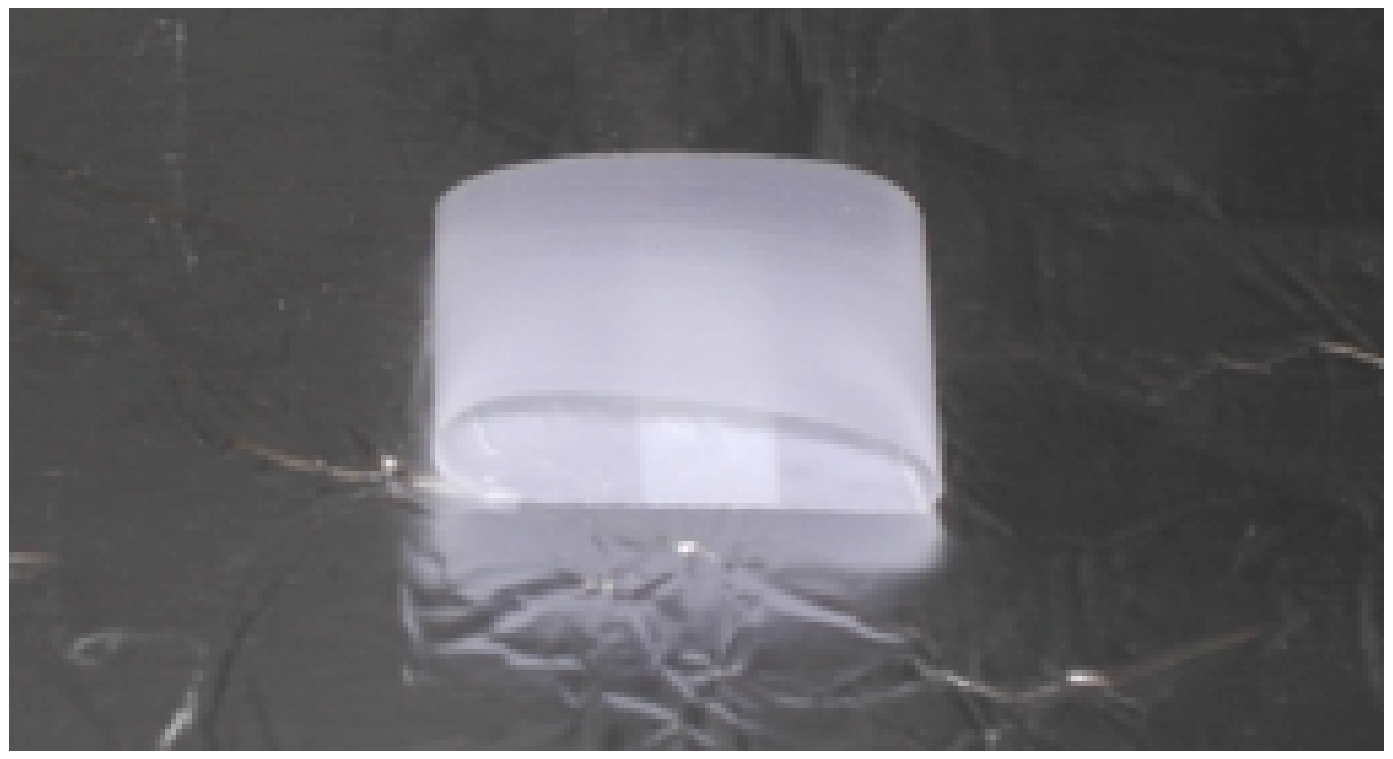

Figure 13: Thin tape curled back on itself to hold cardstock pieces. 


\section{Cosmic Ray Detector}

8. Measure out segments of the black masking tape and stick them onto the scintillator edges (Figure 14). Be careful to wrap the tape tightly around the corners to ensure good adhesion onto the foil and cardstock.

9. Now wrap black masking tape around the joined ends of the photomultiplier and scintillator (Figures 15 and 16). Be sure that all areas of the scintillator are completely covered so that no light leaks through.

Note: If you did not glue the ends of the photomultiplier and scintillator, be extra thorough when taping them together so that they do not come apart during detector assembly. The casing for the cosmic ray detector will help keep the scintillator and photomultiplier in position and alleviate some of the stress on the tape.

10. Set the paddle aside once it is wrapped. Let the scintillator rest on a raised surface (or, conversely, let the photomultiplier sit in a recessed surface). This arrangement will keep strain off the joined ends of the scintillator and photomultiplier, which is especially important if they are not glued together. 


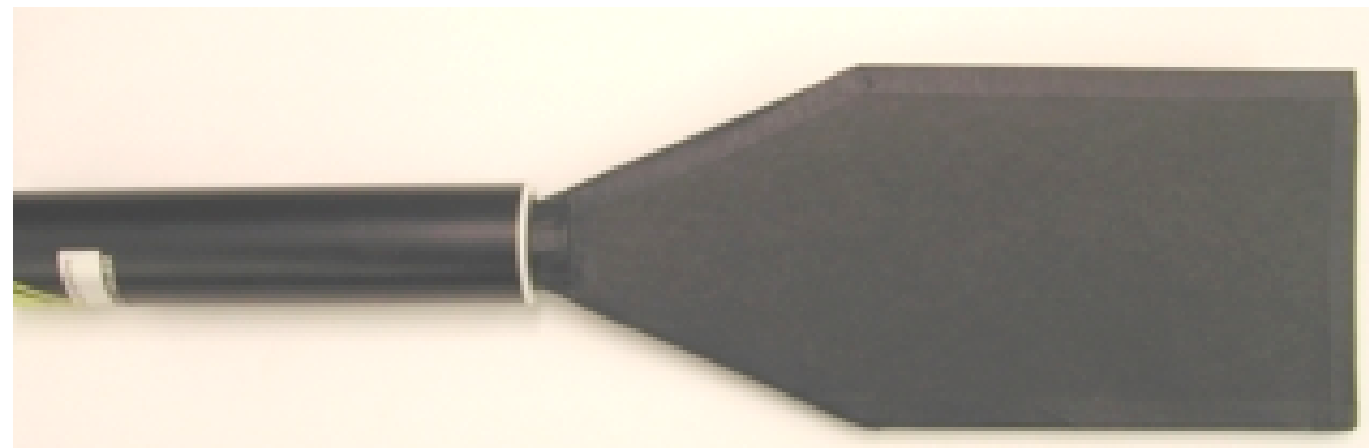

Figure 14: Scintillator with cardstock and taped edges.

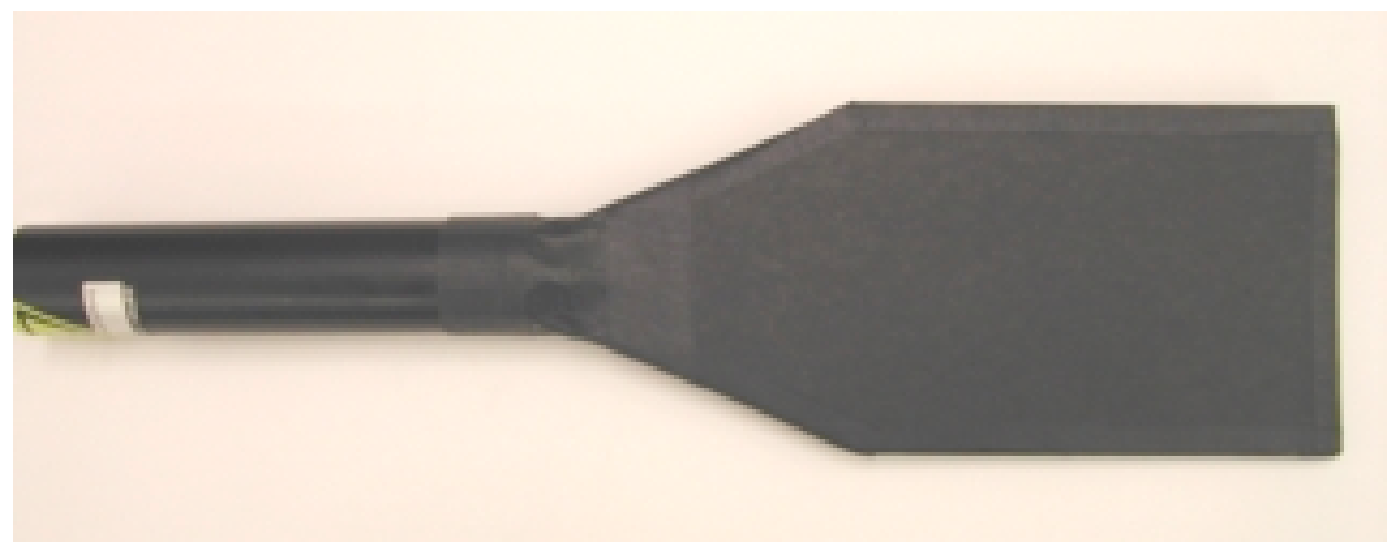

Figure 15: Paddle with interface between scintillator and photomultiplier wrapped in tape.

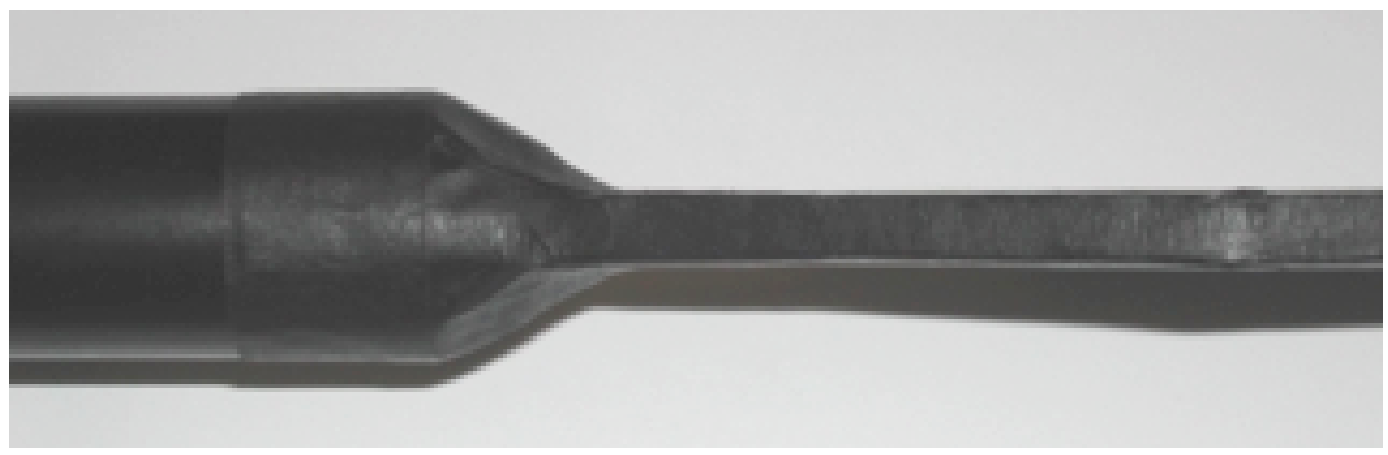

Figure 16: Close-up side view of interface wrapped in tape. 


\section{Cosmic Ray Detector}

\section{Circuit Board}

The circuit board (Figure 17) processes the signals from the photomultiplier tubes, converting them to counts given as numbers on the display and beeps on the buzzer. It consists of a collection of resistors, capacitors, and semiconductor chips (integrated circuits) among other components soldered onto a board (Figure 18). The following sections outline how to assemble these components into a working board.

\section{Soldering}

The components must be attached to the circuit board by soldering. The circuit board is labeled for each component with a letter and number, which appear next to names of the components in the list below.

If this is your first time soldering (or if your technique needs to be refreshed), you may want to practice on some pieces of scrap first. What is important to remember is that both the component wire and the metal pad on the board need to be hot for the solder to bond. Hold the soldering iron tip against both surfaces for about 1-2 seconds, and then feed the solder until the wire and pad are covered. The surfaces are hot enough when the melted solder flows across them on its own - like water saturating a paper towel. Be careful not to let the component get too hot; it is no good if it is melted.

\begin{tabular}{llll} 
Item & Description & $\#$ & Position on Board \\
1 & $51 \square$ resistor & 3 & $\mathrm{R} 1, \mathrm{R} 7, \mathrm{R} 19$ \\
2 & $470 \square$ resistor & 1 & $\mathrm{R} 12$ \\
3 & $1.1 \mathrm{k} \square$ resistor & 2 & $\mathrm{R} 8, \mathrm{R} 20$ \\
4 & $4.7 \mathrm{k} \square$ resistor & 3 & $\mathrm{R} 10, \mathrm{R} 11, \mathrm{R} 14$ \\
\hline 5 & $10 \mathrm{k} \square$ resistor & 4 & $\mathrm{R} 2, \mathrm{R} 16, \mathrm{R} 22, \mathrm{R} 23$ \\
6 & $11 \mathrm{k} \square$ resistor & 4 & $\mathrm{R} 3, \mathrm{R} 6, \mathrm{R} 15, \mathrm{R} 18$ \\
7 & $12 \mathrm{k} \square$ resistor & 4 & $\mathrm{R} 4, \mathrm{R} 9, \mathrm{R} 17, \mathrm{R} 21$ \\
8 & $47 \mathrm{k} \square$ resistor & 1 & $\mathrm{R} 25$ \\
9 & $100 \mathrm{k} \square$ resistor & 1 & $\mathrm{R} 5$ \\
10 & $120 \mathrm{k} \square$ resistor & 1 & $\mathrm{R} 13$ \\
11 & $100 \mathrm{k} \square$ variable resistor & 1 & $\mathrm{R} 24$ \\
12 & $1.2 \mathrm{k} \square$ resistor array & 3 & $\mathrm{RN} 1, \mathrm{RN} 2, \mathrm{RN} 3$ \\
13 & $100 \mathrm{pF}$ capacitor & 2 & $\mathrm{C} 5, \mathrm{C} 11$ \\
14 & $2.2 \mathrm{pF}$ capacitor & 1 & $\mathrm{C} 13$ \\
15 & $0.1 \square \mathrm{F}$ capacitor & 14 & $\mathrm{C} 3, \mathrm{C} 6, \mathrm{C} 7, \mathrm{C} 8, \mathrm{C} 9, \mathrm{C} 10, \mathrm{C} 12, \mathrm{C} 14$, \\
& & & $\mathrm{C} 15, \mathrm{C} 17, \mathrm{C} 18, \mathrm{C} 19, \mathrm{C} 20, \mathrm{C} 21$
\end{tabular}

\section{Equipment and Supplies}

- Soldering iron

- Solder (medium diameter)
- Wet sponge

- Circuit Board 


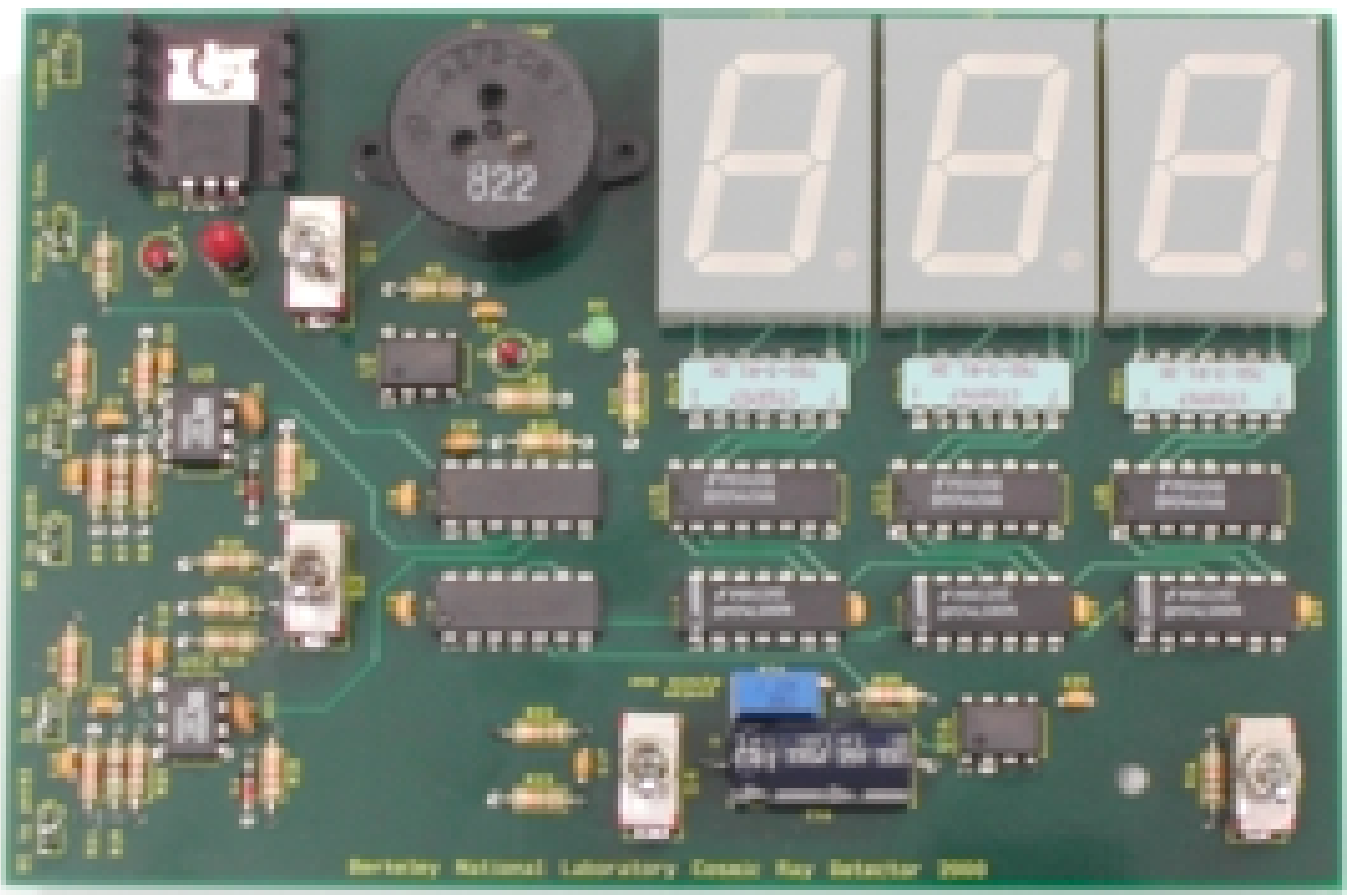

Figure 17: Completed circuit board.

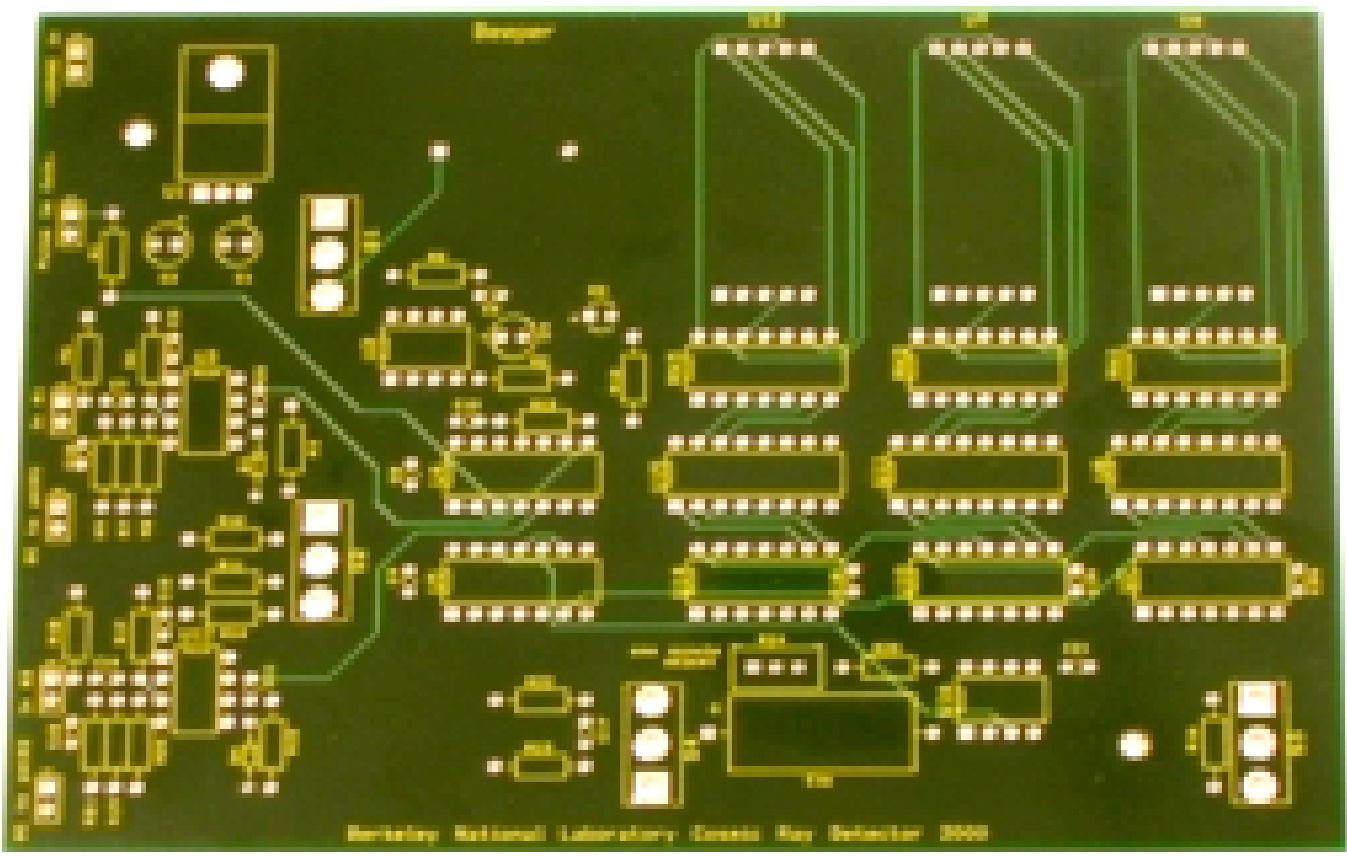

Figure 18: Empty circuit board. 


\section{Cosmic Ray Detector}

\begin{tabular}{|c|c|c|c|}
\hline Item & Description & Quantity & Position on Board \\
\hline 16 & $1 \square$ F capacitor & 1 & $\mathrm{C} 4$ \\
\hline 17 & $6.8 \square \mathrm{F}$ capacitor & 1 & $\mathrm{C} 2$ \\
\hline 18 & $33 \square \mathrm{F}$ capacitor & 1 & $\mathrm{C} 1$ \\
\hline 19 & $330 \square \mathrm{F}$ capacitor & 1 & $\mathrm{C} 16$ \\
\hline 20 & Diode & 2 & D1, D4 \\
\hline 21 & LED (green) & 1 & D3 \\
\hline 22 & Buzzer & 1 & D2 \\
\hline 23 & IC precision timer & 2 & $\mathrm{U} 2, \mathrm{U} 16$ \\
\hline 24 & IC 7 ns comparator & 2 & U3, U12 \\
\hline 25 & IC 2-in and gate & 1 & U4 \\
\hline 26 & IC QUAD 2-in $n$ and gate & 1 & U5 \\
\hline 27 & IC Decade counter & 3 & U7, U10, U14 \\
\hline 28 & IC BCD-to-SEG decoder & 3 & U8, U11, U15 \\
\hline 29 & Large LED & 3 & U6, U9, U13 \\
\hline 30 & $5 \mathrm{~V}$ regulator & 1 & U1 \\
\hline 31 & Switch on-on & 2 & $\mathrm{~S} 1, \mathrm{~S} 3$ \\
\hline 32 & Switch on-off-on & 2 & $\mathrm{~S} 2, \mathrm{~S} 4$ \\
\hline 33 & 2 Pin header male & 6 & JP1, JP2, JP3, JP4, JP5, JP6 \\
\hline
\end{tabular}

\section{Procedure}

Although the order of assembly is not essential, you may want to start with the small pieces first and work your way up to the larger pieces. This order makes holding onto the pieces easier - they just lie flat against the table. Additionally, you may want to start with the resistors if you are unsure of your skill; resistors are less sensitive to damage by heat and provide good practice before moving on to more delicate components such as integrated circuits (IC).

1. Locate $R 1$ on the circuit board and insert the $51 \square$ resistor (Item 1) into it (Figure 19). The resistor should sit on the label side of the board. Be sure to bend the wires slightly on the other side such that the resistor will hold in place. See Appendix B for color codes on resistors.

2. Turn the board to its backside and place the hot soldering iron tip to both the resistor wire and the metal pad on the board.

3. Once the surfaces are hot (after $\sim 1-2$ seconds), feed the solder to them until they are covered.

4. Repeat step 3 for the other wire.

5. Trim the excess wire on the resistor. Try to keep the wire about $1-2 \mathrm{~mm}$ long.

6. Repeat the above steps for other components. See Figure 20 for resistors. 


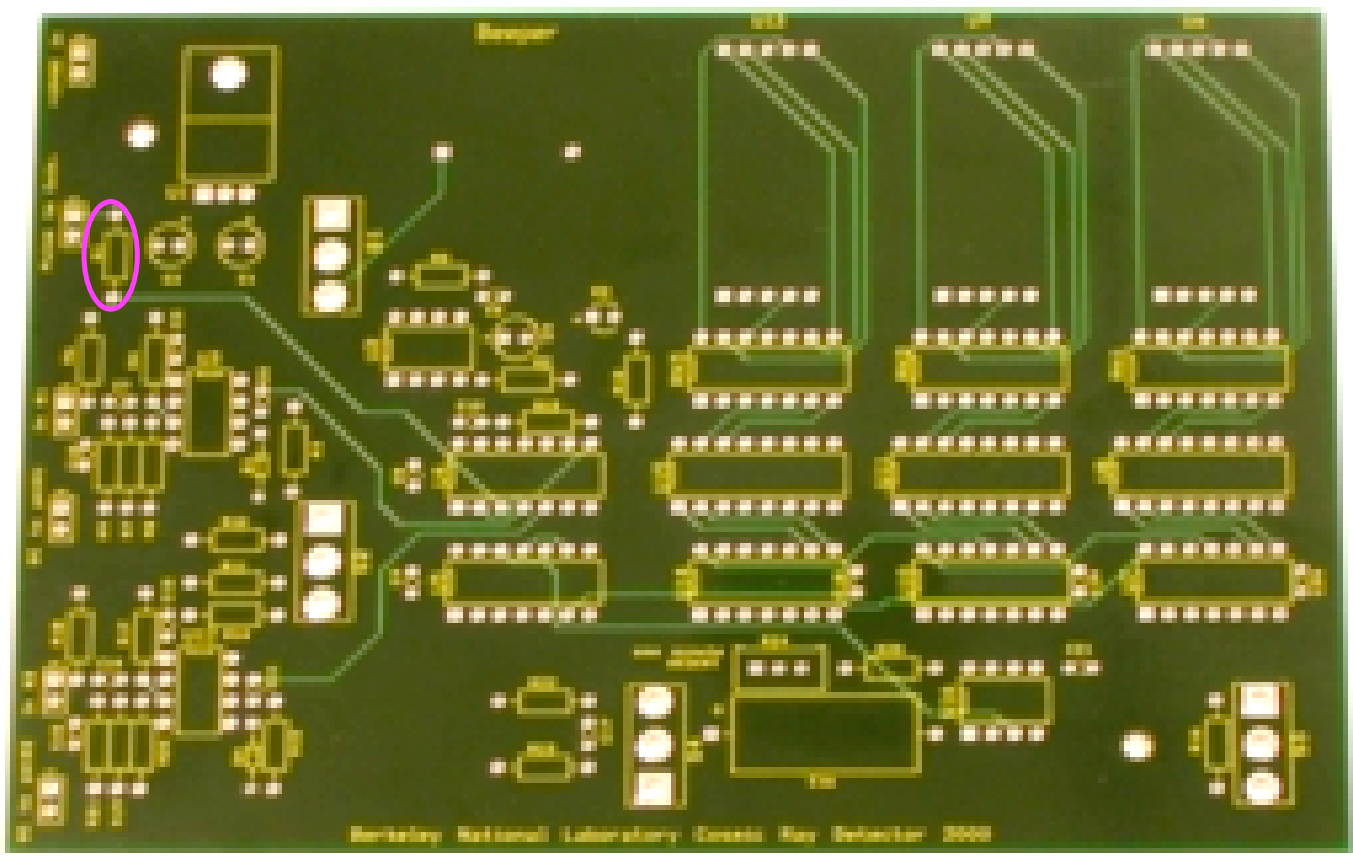

Figure 19: Circuit board with Rl highlighted.

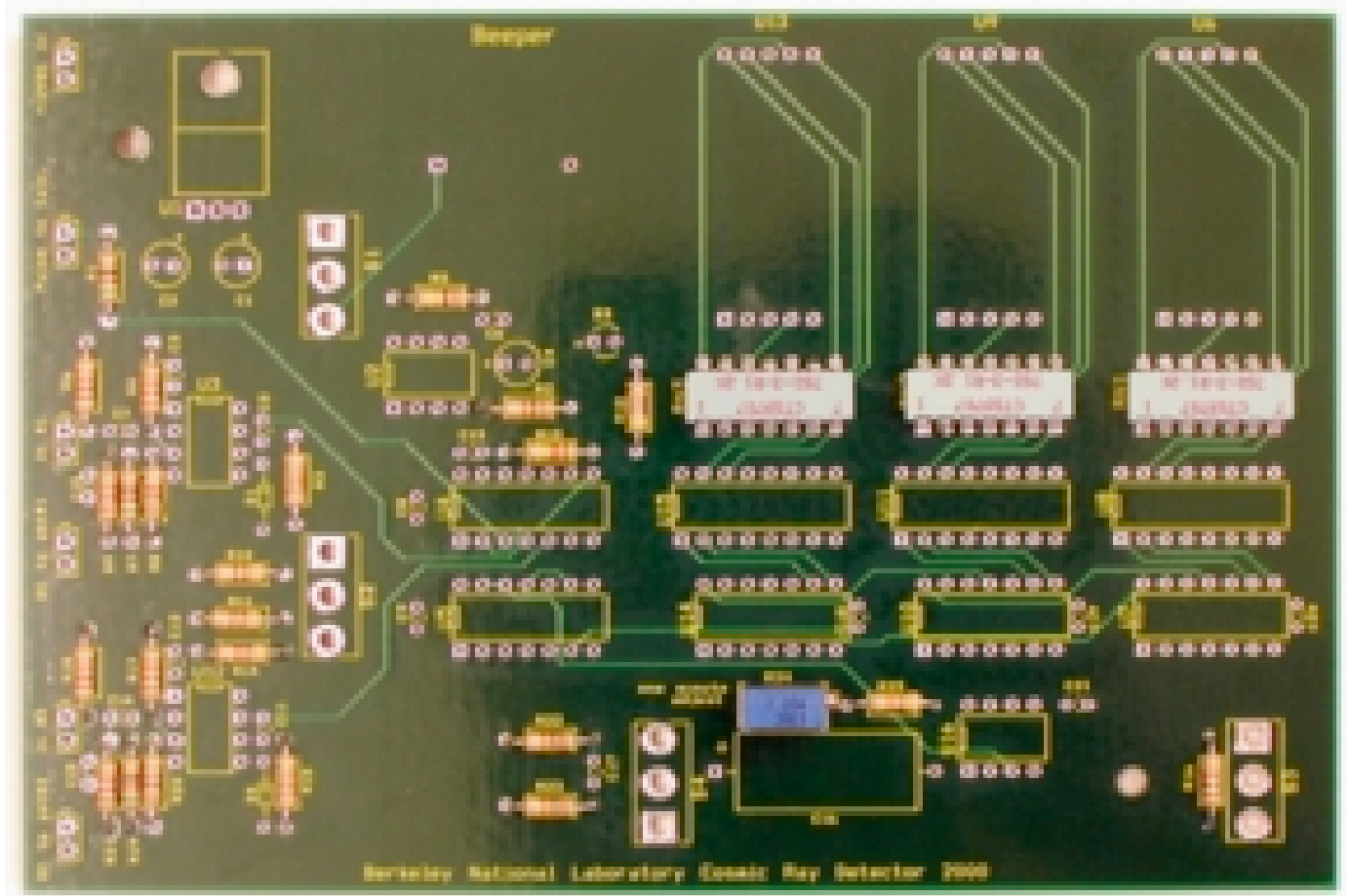

Figure 20: Circuit board with all resistors in place. 
Note on Resistors: For the variable resistor (Item 11), be sure that the adjustment screw faces rightward when looking at the board with its main label right side up.

For the resistor arrays (Item 12), be sure that they are in the right orientation. If you look at the writing right side up, then the upper right corner has an approximately square protrusion. This protrusion should be aligned with the square metal pad on the board.

Note on Capacitors: Items 16-18 ( $1 \square \mathrm{F}, 6.8 \square \mathrm{F}$, and $33 \square \mathrm{F}$ capacitors) have a particular orientation: align the + sign on the capacitor with the + sign on the board (corresponding to the square pad).

On Item 19 (330 $\square$ F capacitor) is marked an arrow that points in the negative lead direction. Place this capacitor such that the base of the arrow aligns with the $+\operatorname{sign}($ square pad). See Figure 21.

Note on Diodes: Item 20 (diode) has a specific orientation: the band on the diode corresponds to the band labeled on the board.

Item 21 (LED) should be placed such that the longer wire inserts into the square pad (labeled with + sign). See Figure 21.

Note on Integrated Circuits: Items 23-28 (integrated circuits) have specific orientations also. The dot in one of the corners should align with the square pad on the board. Some of them also have bands or notches or both. These bands and notches correspond to the square notches drawn on the board. They all have dots though, so you do not have to worry about the bands and notches. See Figure 22.

Note on 5 V Regulator: Item 30 (5 V regulator) has a definite orientation. With the lettering facing you, the left-most lead is "in" the middle is "ground" and the right most is "out." Power from the power supply needs to go into the "in" lead, which should align with the square pad on the board. Although not necessary, a heat sink (as shown in Figure 22) is preferred. Simply place the heat sink on the board, bend the $5 \mathrm{~V}$ regulator overtop it, and fasten them together with a nut and bolt.

Note on Large LED: Item 29 (large LED) should be placed such that the decimal point is in the lower right corner when looking at the board with the main label right side up.

Note on Switches: Items 31 and 32 (switches) should be carefully distinguished. Be sure not to mix them; their orientations, however, do not matter. 


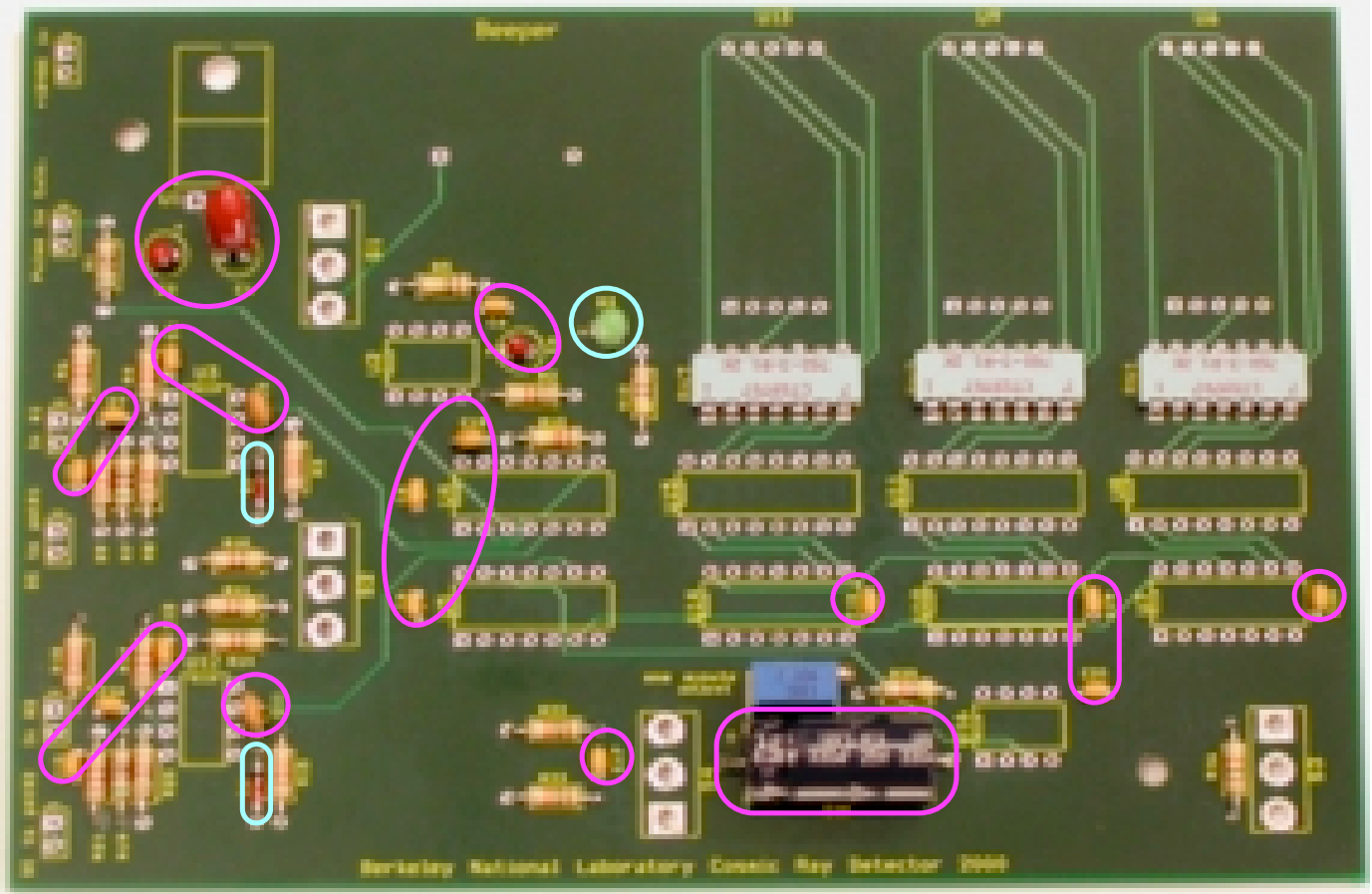

Figure 21: Circuit board showing highlighted capacitors (pink) and diodes (aqua).

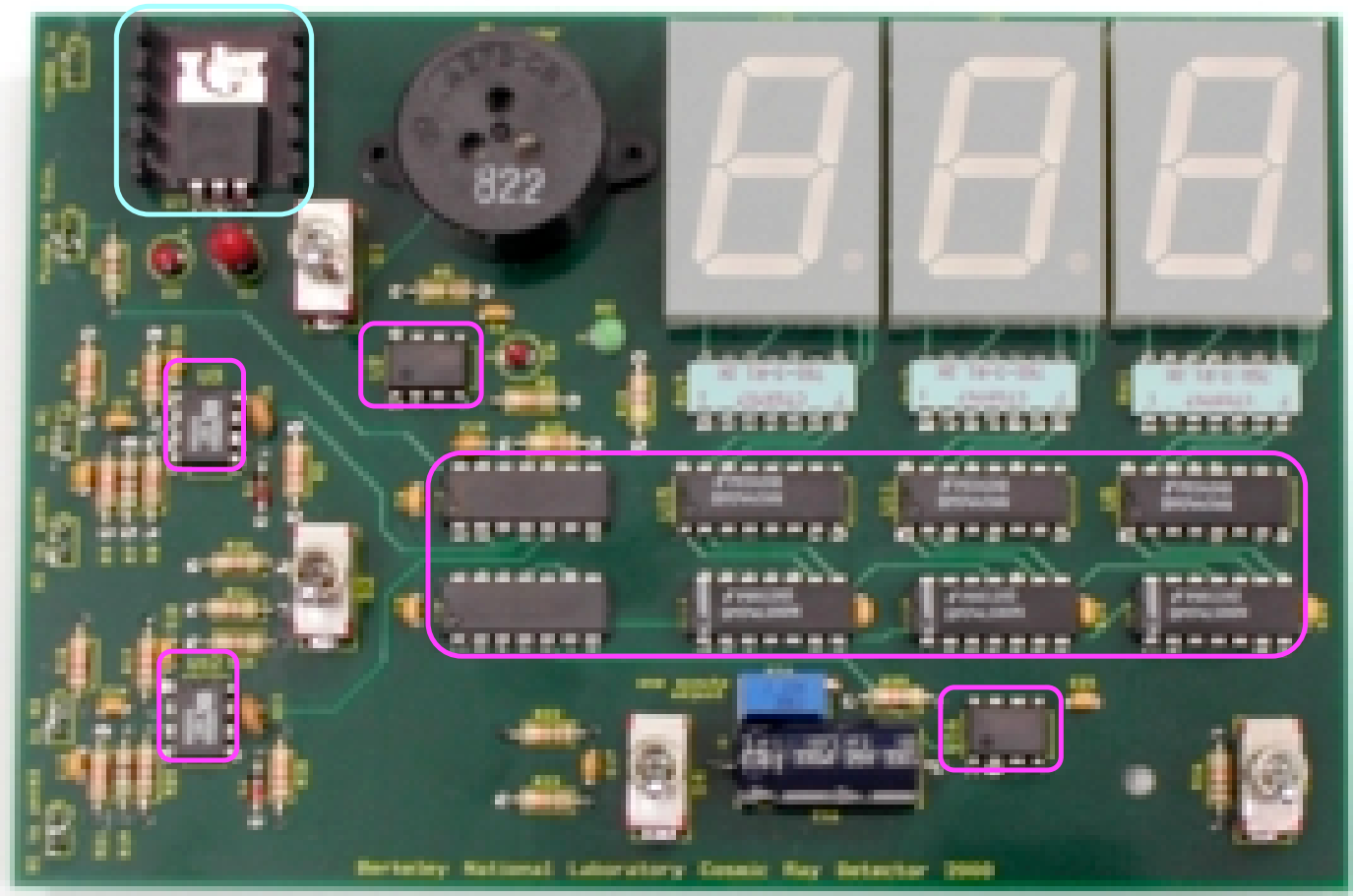

Figure 22: Completed circuit board showing highlighted integrated circuits (pink) and $5 \mathrm{~V}$ regulator with heat sink (aqua). 


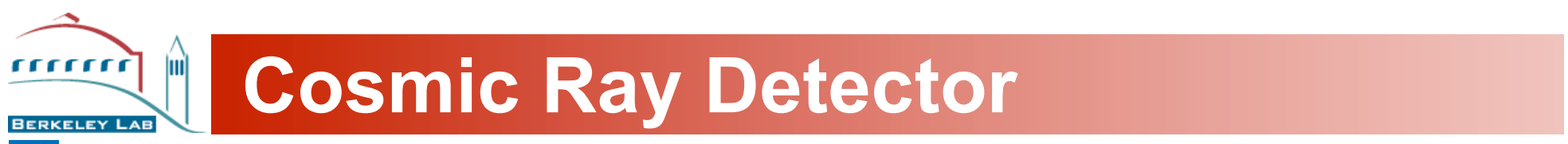

Note on 2 Pin Header: Item 33 ( 2 pin header) should be placed on the underside of the board. The 2 pins should face the outer edge of the board while the polarizing plastic protrusion should face toward the inside of the board as shown in Figure 23. Solder the pins from the topside of the board. 


\section{Assembly Manual}
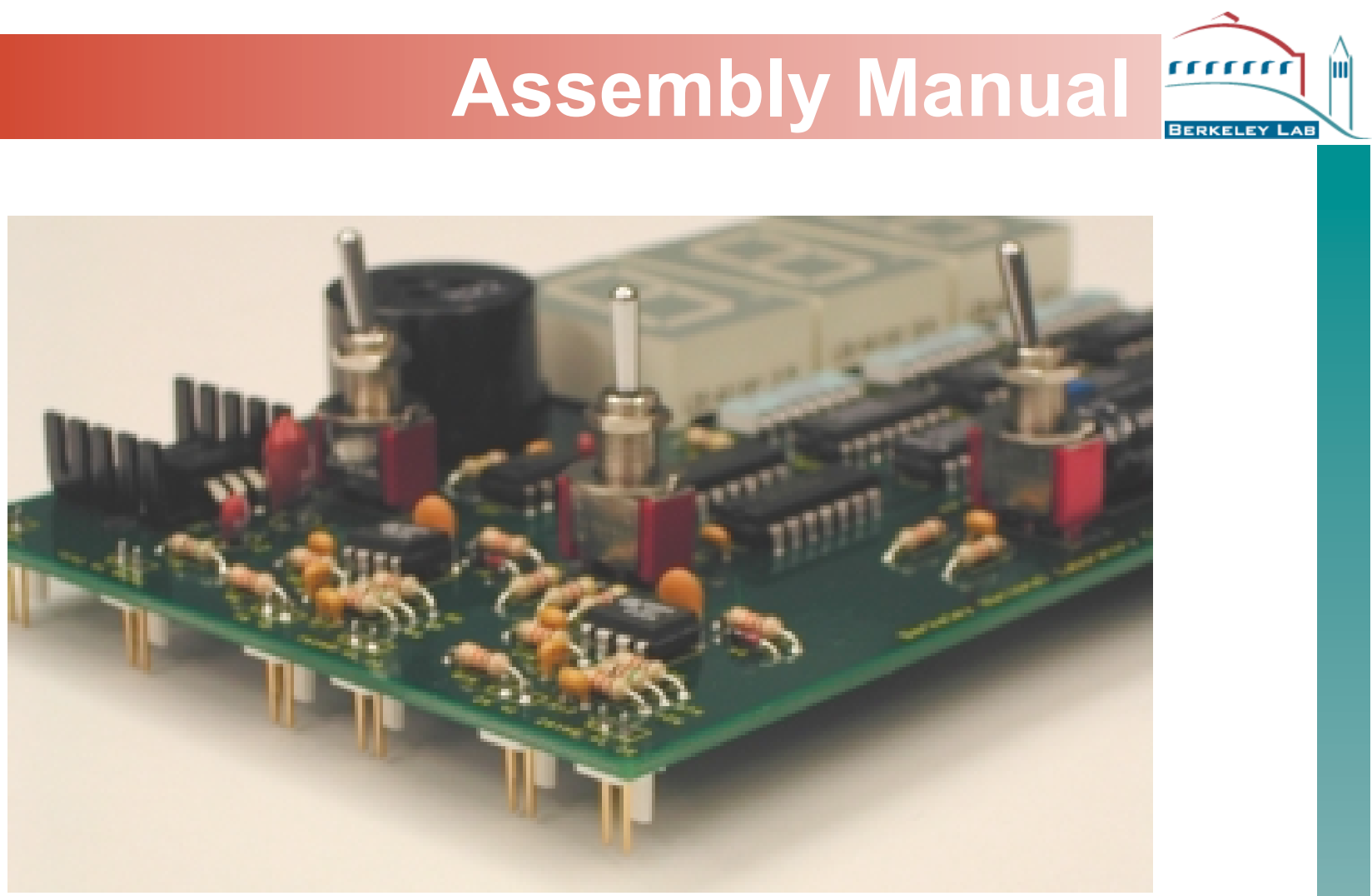

Figure 23: Side view of circuit showing placement of 2 pin headers. 


\section{Cosmic Ray Detector}

\section{Casing}

The casing is important for holding together the components of the detector (Figure 24); the casing serves as an exoskeleton. You may design your own or you can follow the Berkeley Lab model (see Appendix C). If you choose to build your own design, keep in mind the requirements described below.

The spacing and orientation of the scintillator paddles are specific. The paddles must be parallel to each other and separated by 4 inches as measured from between the 2 surfaces (Figure 25). Also, the casing itself should have a sturdy opening between the gap of the scintillator paddles (as shown in Figure 24). This opening allows heavy objects (such as bricks and lead blocks) to be placed between the paddles, which is part of a couple of experiments.

When designing your casing, be sure to provide adequate support for the scintillator paddles at both the scintillator part and the photomultiplier part. This 2-part support keeps strain off the interface between them.

The circuit board is the other component that needs to be attached to the casing. It should be placed such that the switches are accessible and that the LED display is clearly visible. Also, it has a variable resistor (that controls the length of a timed count) that may need to be adjusted. Be sure to allow access to the resistor. In the Berkeley Lab design (Figure 24), the circuit board is mounted onto a front panel of Lucite (bolted by the switches themselves).

Additionally, you should consider the power and data cables that need to pass through the casing. Though the choice is yours, you can extend the life of the circuit board and add convenience to using the detector by attaching connectors to the casing. The connectors allow you to plug in power and data cables without tugging on the circuit board (especially during transportation). Also, they allow interchanges of standard cables for wall transformers and CBL data cords. (This is useful for instances in which the initial wall transformer or CBL cord stops working or is misplaced.) 


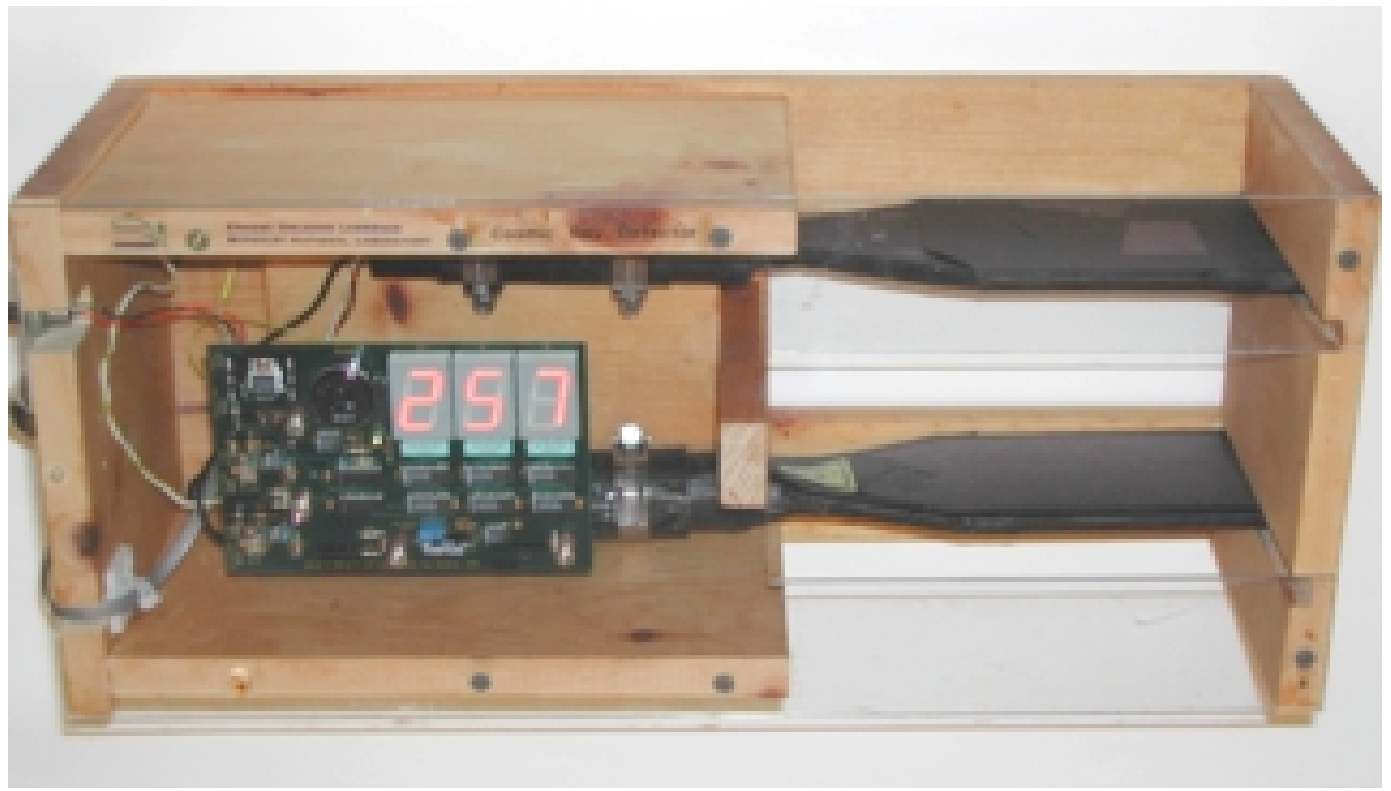

Figure 24: Completed cosmic ray detector.

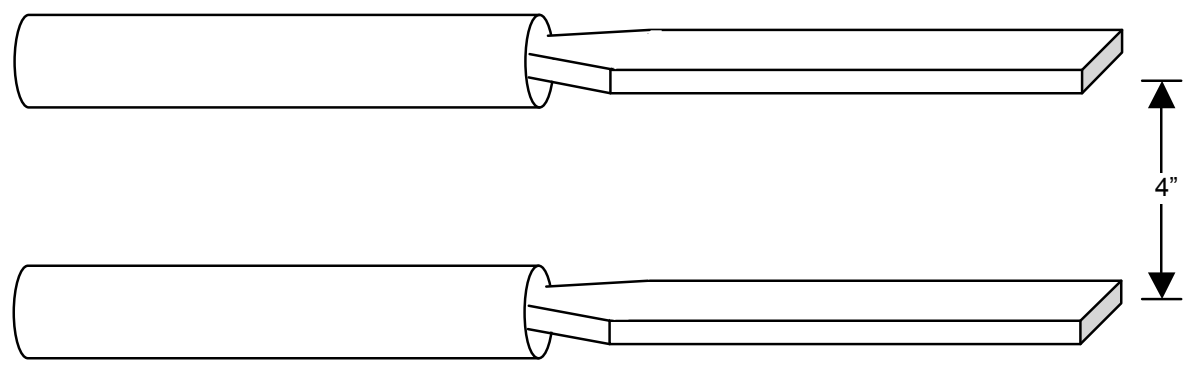

Figure 25: Illustration of separation between scintillator paddles. 
Wiring

Once the electronic parts of the detector are mounted to the casing, they must be connected to each other with wires. The 2 pin headers on the circuit board allow the wires to be plugged in rather than soldered. Because these are polar headers, you must be careful to distinguish your hot and ground wires appropriately when making the corresponding plugs.

\section{Equipment and Supplies}

- Wire stripper

- Wire cutter

- Electrical tape

- Solder

- Soldering iron
- Moist sponge

- 2 pin header connectors

- Crimp female pins

- Crimp tool

- CBL cable

\section{Procedure}

1. Strip the end of the wire that will attach to the board using the wire stripper. Be sure to strip enough away such that the exposed wire makes good contact with the crimp pin.

2. Tin the stripped end of the wire. That is, first twist the frayed end with your fingers to make it tight, and then lightly solder the twisted end. Be sure to keep the wire thin to fit into the crimp pin.

3. Insert the tinned wire into the crimp pin as far as it goes and place the pin in the crimp tool. This may be awkward and frustrating depending on your skill and the crimp tool you use. You may want to place the pin in the crimp tool first and then insert the wire. Do what works best for you.

4. Crimp. Again, this may become frustrating; you may have to try this several times before you master it.

5. Repeat for all the other wires. However, if your photomultiplier has additional wires for setting the gain, do not crimp them. Instead, short the gain wires by twisting them together, soldering them to each other, and then wrapping tape around the exposed ends (Figure 26).

6. Insert crimp pins into 2-pin header connector as far as they go. You may feel a click; they should not pull out (Figure 27).

Note: Be sure to insert the pins in the correct orientation. That is, when plugged into the board, ground should match with ground on the board (circular metal pad) and hot / signal should match with hot / signal on the board (square metal pad). Ground is typically black while hot is typically white or red. 


\section{Assembly Manual}
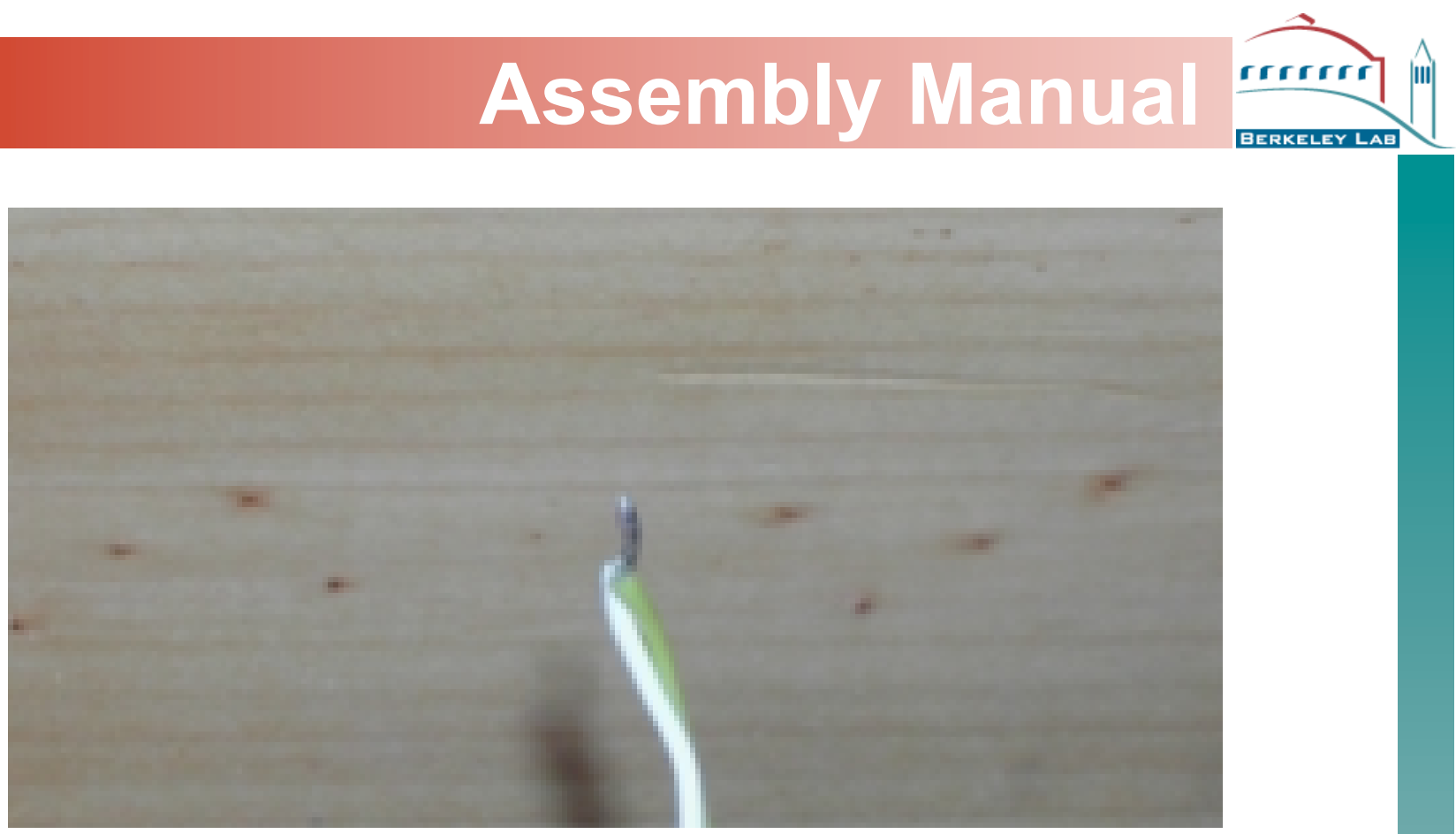

Figure 26: Gain wires shorted.

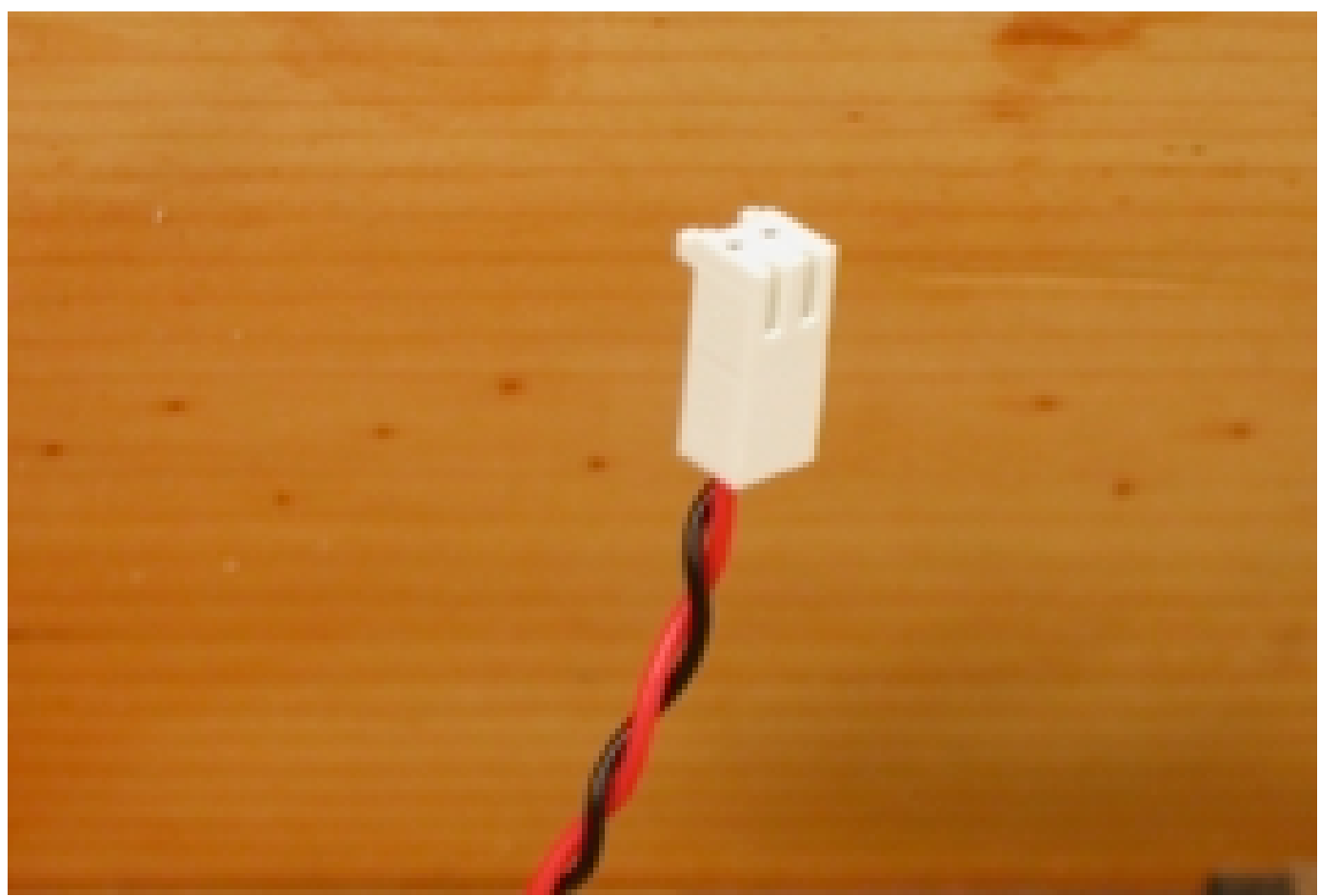

Figure 27: Power wires attached to 2-pin header connector. 


\section{Cosmic Ray Detector}

\section{Appendix A: Suppliers and Parts}

The following subsections list certain parts available from some suppliers. Berkeley Lab does not endorse these suppliers but offers their names for the sake of convenience. In this manual we use photodetector package P30CW5. We are currently testing a less expensive side mount tube.

\section{Scintillator Paddles}

\begin{tabular}{|c|c|c|c|c|}
\hline Part & Quantity & Supplier & Phone Number & Web Address \\
\hline \multirow{2}{*}{ Scintillator } & \multirow{2}{*}{$\begin{array}{l}1-10 " \times 12 " X \\
1 / 4 \text { " sheet }\end{array}$} & Bicron & (440) 564-2251 & www.bicron.com \\
\hline & & Eljen Technology & (888) 800-8771 & www.eljentechnology.com \\
\hline Optical Cement & $250 \mathrm{ml}$ & (same as above) & & \\
\hline Photomultiplier & 2 & Electron Tubes Ltd. & (800) 521-8382 & www.electrontubes.com \\
\hline
\end{tabular}

\section{Circuit Board}

Please contact Dr. Howard Matis at (510) 486-5031 or HSMatis@lbl.gov for information on obtaining the circuit board. The circuit board components can be purchased from Digi-Key at 1800 DIGI-KEY or www.digi-key.com. Please note that one part must be purchased from Newark Electronics - 18002 NEWARK.

A parts list is on the next page. In addition to those parts, you also need a wall transformer that supplies $800 \mathrm{~mA}$ at $12 \mathrm{~V} \mathrm{DC}$. These transformers are common and can be found at any electronics store. Be sure to get the corresponding connector for the casing if you choose to use one. 


\begin{tabular}{|c|c|c|c|c|c|}
\hline Item & $\begin{array}{l}\text { Parts* } \\
\text { List \# }\end{array}$ & Description & $\#$ & Digi-Key Part & Position on Board \\
\hline 1 & 13 & $51 \square$ resistor & 3 & 51QBK-ND & $\mathrm{R} 1, \mathrm{R} 7, \mathrm{R} 19$ \\
\hline 2 & 14 & $470 \square$ resistor & 1 & 470QBK-ND & $\mathrm{R} 12$ \\
\hline 3 & 15 & $1.1 \mathrm{k} \square$ resistor & 2 & 1.1KQBK-ND & $\mathrm{R} 8, \mathrm{R} 20$ \\
\hline 4 & 16 & $4.7 \mathrm{k} \square$ resistor & 3 & 4.7KQBK-ND & R10, R11, R14 \\
\hline 5 & 17 & $10 \mathrm{k} \square$ resistor & 4 & 10KQBK-ND & R2, R16, R22, R23 \\
\hline 6 & 18 & $11 \mathrm{k} \square$ resistor & 4 & $11 \mathrm{KQBK}-\mathrm{ND}$ & R3, R6, R15, R18 \\
\hline 7 & 19 & $12 \mathrm{k} \square$ resistor & 4 & 12KQBK-ND & R4, R9, R17, R21 \\
\hline 8 & 20 & $47 \mathrm{k} \square$ resistor & 1 & 47KQBK-ND & $\mathrm{R} 25$ \\
\hline 9 & 21 & $100 \mathrm{k} \square$ resistor & 1 & 100KQBK-ND & R5 \\
\hline 10 & 22 & $120 \mathrm{k} \square$ resistor & 1 & 120KQBK-ND & $\mathrm{R} 13$ \\
\hline 11 & 23 & $100 \mathrm{k} \square$ variable resistor & 1 & 3296X-104-ND & R24 \\
\hline 12 & 12 & $1.2 \mathrm{k} \square$ resistor array & 3 & 760-3-R1.2K-ND & RN1, RN2, RN3 \\
\hline 13 & 1 & $100 \mathrm{pF}$ capacitor & 2 & P4037A-ND & $\mathrm{C} 5, \mathrm{C} 11$ \\
\hline 14 & 2 & $2.2 \mathrm{pF}$ capacitor & 1 & 1119PHCT-ND & $\mathrm{C} 13$ \\
\hline 15 & 3 & $0.1 \square \mathrm{F}$ capacitor & 14 & $399-2155$ & $\begin{array}{l}\mathrm{C} 3, \mathrm{C} 6, \mathrm{C} 7, \mathrm{C} 8, \mathrm{C} 9, \\
\mathrm{C} 10, \mathrm{C} 12, \mathrm{C} 14, \mathrm{C} 15, \\
\mathrm{C} 17, \mathrm{C} 18, \mathrm{C} 19, \mathrm{C} 20, \\
\mathrm{C} 21\end{array}$ \\
\hline 16 & 4 & $1 \square \mathrm{F}$ capacitor & 1 & P2105-ND & $\mathrm{C} 4$ \\
\hline 17 & 5 & $6.8 \square \mathrm{F}$ capacitor & 1 & P2037-ND & $\mathrm{C} 2$ \\
\hline 18 & 6 & $33 \square$ F capacitor & 1 & P2052-ND & $\mathrm{C} 1$ \\
\hline 19 & 7 & $330 \square \mathrm{F}$ capacitor & 1 & 4036РHCT & $\mathrm{C} 16$ \\
\hline 20 & 8 & Diode & 2 & 1N4148MSCT & D1, D4 \\
\hline 21 & 10 & LED (green) & 1 & 160-1080-ND & D3 \\
\hline 22 & 9 & Buzzer & 1 & P9948-ND & D2 \\
\hline 23 & 27 & IC precision timer & 2 & 296-1411-5-ND & $\mathrm{U} 2, \mathrm{U} 16$ \\
\hline 24 & 28 & IC 7 ns comparator & 2 & $48 F 1889 \dagger$ & U3, U12 \\
\hline 25 & 29 & IC $2-I N$ and gate & 1 & DM7408N-ND & U4 \\
\hline 26 & 30 & IC QUAD 2-in $\mathrm{n}$ and gate & 1 & DM74LS00N-ND & U5 \\
\hline 27 & 32 & IC Decade counter & 3 & DM74LS90N-ND & U7, U10, U14 \\
\hline 28 & 33 & IC BCD-to-SEG decoder & 3 & DM7447AN-ND & U8, U11, U15 \\
\hline 29 & 31 & Large LED & 3 & 67-1485-ND & U6, U9, U13 \\
\hline 30 & 26 & $5 \mathrm{~V}$ regulator & 1 & LM340T-5.0-ND & U1 \\
\hline 31 & 24 & Switch on-on & 2 & CKN1021-ND & $\mathrm{S} 1, \mathrm{~S} 3$ \\
\hline 32 & 25 & Switch on-off-on & 2 & CKN1025-ND & $\mathrm{S} 2, \mathrm{~S} 4$ \\
\hline 33 & 11 & 2 Pin header & 6 & ED7202-ND & $\begin{array}{l}\text { JP1, JP2, JP3, JP4, } \\
\text { JP5, JP6 }\end{array}$ \\
\hline 34 & & 2 Pin header connector & 6 & A19111-ND & $\begin{array}{l}\text { Connects with Item } \\
33\end{array}$ \\
\hline
\end{tabular}

*For a current version go to http://www.lbl.gov/abc/cosmic/

Digi-Key - http://www.DigiKey.com

Newark Electronics $\dagger-$ http://www.Newark.com (part 24) 


\section{Cosmic Ray Detector}

\section{Appendix B: Resistor Color Codes}

3 colored bands and possibly a gold or silver band code each resistor, which identify the resistance in ohms. The first 2 bands (starting from the farthest on the left - opposite the gold or silver band if present) indicate the first 2 digits of the resistance. The third band indicates the multiplier of 10. The color codes are

$\begin{array}{ll}\text { Black } & 0 \\ \text { Brown } & 1 \\ \text { Red } & 2 \\ \text { Orange } & 3 \\ \text { Yellow } & 4 \\ \text { Green } & 5 \\ \text { Blue } & 6 \\ \text { Violet } & 7 \\ \text { Gray } & 8 \\ \text { White } & 9\end{array}$

For example, a resistor coded blue-red-orange is $6-2-3$, which stands for $62 \square 10^{3}$ ohms (or $62 \mathrm{k} \square$ ).

Usually, the resistor also has a fourth band — the gold or silver one. This band indicates the tolerance of the stated resistance: gold stands for $1 \%$, silver for $5 \%$, and no fourth band for $20 \%$. 


\section{Appendix C: Optional Dimensions for Casing}

The table that follows Figure 28 lists pieces and their dimensions for the casing developed at Berkeley Lab. Figure 28 shows the casing with numbered pieces that correspond to the numbered pieces of the table. (The metal brackets shown in the picture hold the photomultipliers.)

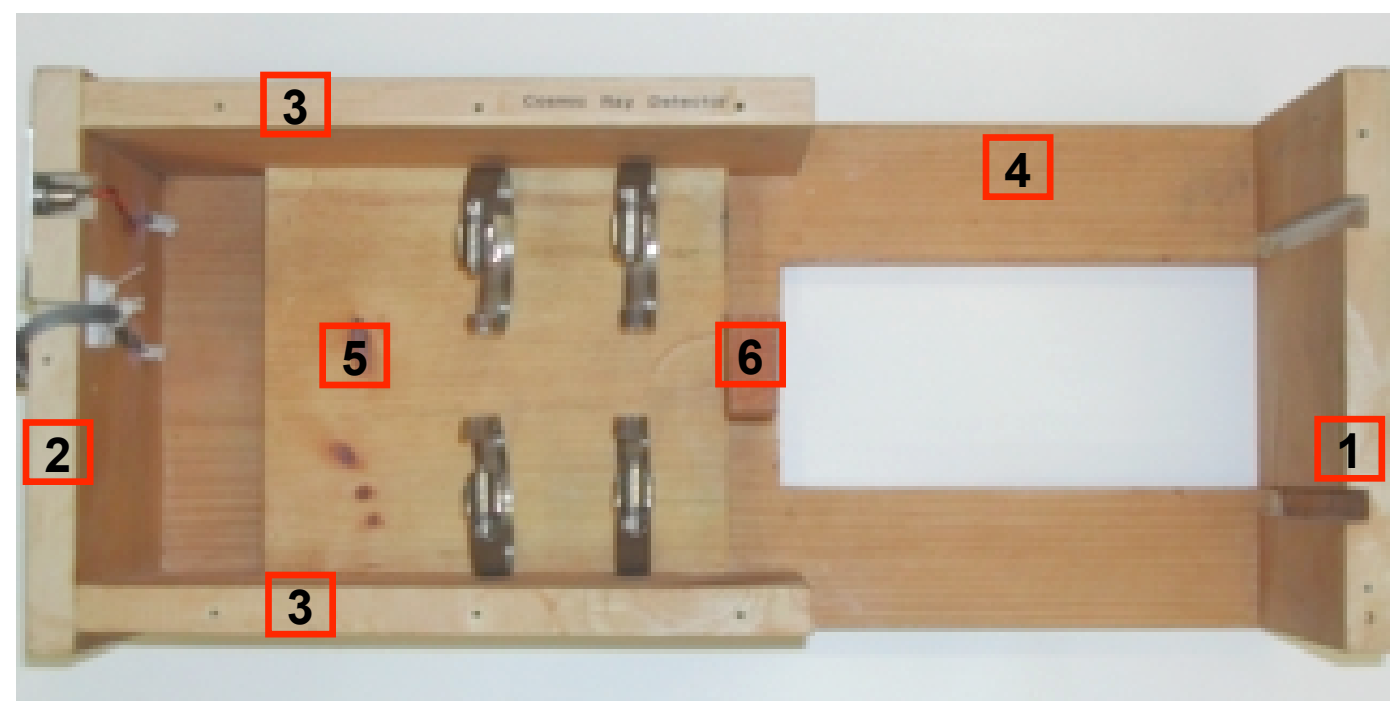

Figure 28: Berkeley Lab casing with pieces numbered.

\begin{tabular}{|c|c|c|c|c|}
\hline Piece & Quantity & Width [inches] & Length [inches] & Thickness [inches] \\
\hline 1 & 1 & 6.5 & 9 & 0.75 \\
\hline 2 & 1 & 6.5 & 9 & 0.75 \\
\hline 3 & 2 & 6.5 & 11.25 & 0.75 \\
\hline 4 & 1 & 9 & 21 & 0.75 \\
\hline 5 & 1 & 7 & 8 & 1.5 \\
\hline 6 & 1 & 1.5 & 6.5 & 0.75 \\
\hline
\end{tabular}

The grooves in Piece 1 are 0.375 inches deep and 0.5 inches wide. They are 2.5 inches from the top and bottom edges of the board and 4 inches apart from each other. Piece 4 and the Lucite front panel have the same shape and special dimensions shown in Figure 29.

You may want to decrease the cutout for Piece 4 to 3" (instead of 4"), so that items can be supported on the plastic and wood backing. This ledge is useful to place absorbers between the scintillators. This ledge is useful for supporting heavy absorbing objects such as clay or lead bricks between the scintillator paddles. 


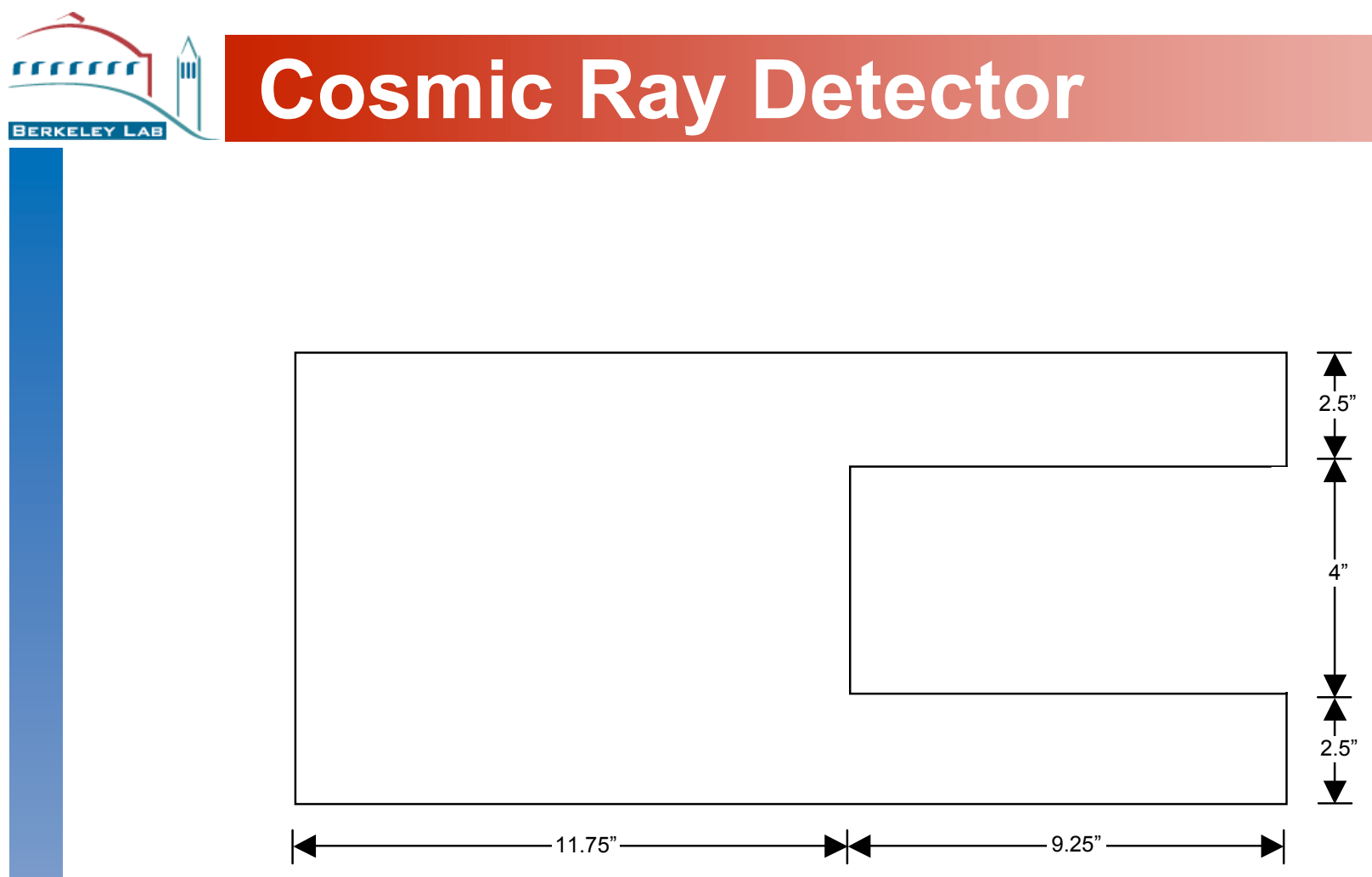

Figure 29: Dimensions for Piece 4 and Lucite front panel. 


\section{Appendix D: Acknowledgements}

We welcome corrections and improvements to this manual. Please send them to Howard Matis - hsmatis@lbl.gov.

The William F. and Edith R. Meggers Project Award from the American Institute of Physics helped in large part to fund the Berkeley Lab Cosmic Ray Detector. Additional support came from the Department of Energy, Center for Science and Engineering at Lawrence Berkeley National Laboratory, and the Energy Research Undergraduate Laboratory Fellowship.

This manual is a revised and expanded version of LBNL-46911, which was written by Colleen Twitty and Howard Matis.

This manuscript was prepared for the US Department of Energy under contract number DE-AC0-76SF00098. 